\title{
(Un)doing gender empirisch erforschen
}

\author{
Julia C. Nentwich und Franziska Vogt
}

\subsection{Konzeptionelle und empirische Herausforderungen}

Sozialkonstruktivistische Konzeptionen von Geschlecht haben seit über 30 Jahren Konjunktur in den Sozialwissenschaften (Gildemeister und Wetterer 1992). Der von West \& Zimmerman bereits 1987 geprägte Begriff doing gender ist dabei zum Platzhalter oder ",catch-all-Begriff“ (Westheuser 2015) geworden. Doing gender hat Geschlecht konsequent in der Interaktion verortet und aus dem Individuum herausgeholt. Grundlegend für heutige Konzeptionen von Geschlecht als sozialer und diskursiver Praxis sind hierbei die in der Ethnomethodologie und dem symbolischen Interaktionismus verankerten Konzeptionen von doing gender durch West und Zimmerman (1987), sowie die nur kurze Zeit später publizierte poststrukturalistische Version Butlers (1991/1990). Im Mittelpunkt der Auseinandersetzung steht die Frage, wie unser Handeln (doing) institutionalisiertes Wissen aufgreift und verfestigt, wie es aber auch infrage gestellt und verändert werden kann. Praxistheoretische Zugänge wie die der Soziologinnen Gherardi, Bruni, Poggio (1994, 1995, 2019; Bruni et al. 2004, 2005) und Martin (2001, 2003, 2004) haben ethnomethodologische Konzepte unter Aufgreifen des Butlerschen Performativitätsbegriff weiterentwickelt und so um eine institutionelle Perspektive erweitert.

J. C. Nentwich ( $\square)$

Universität St. Gallen, St. Gallen, Schweiz

E-Mail: julia.nentwich@unisg.ch

F. Vogt

Pädagogische Hochschule St, Gallen, St. Gallen, Schweiz

E-Mail: franziska.vogt@ phsg.ch 
Mit doing gender wurde insbesondere das Verhältnis von Subjekt und gesellschaftlichen oder organisationalen Struktur(en) und Normen neu verstanden. Geschlecht ist in einem sozialkonstruktivistischen Verständnis nichts, das im Individuum liegend über alle Zeit hinweg gegeben ist, sondern das situativ in Interaktion mit anderen Individuen, aber auch den gesellschaftlichen Strukturen sowie kulturell-historisch etablierten Normen hergestellt werden muss. Während die ethnomethodologische Variante zunächst die fortlaufende Rekonstruktion des traditionell schon Vorhandenen in den Mittelpunkt der Analyse rückt - wie also dieselben Normen der Zweigeschlechtlichkeit immer wieder herangezogen werden und $\mathrm{zu}$ immer denselben Zuschreibungen führen - wurde durch Butlers Ausführungen die in jeder Interaktion immer auch bestehende Möglichkeit der Subversion stärker beleuchtet.

Die Situation ist also immer paradox: während Geschlecht situativ einen potenziell fluide-flüchtigen Charakter hat, verweist die Herstellung der Geschlechterdifferenz jedoch auch immer über den situativen Interaktionszusammenhang zeitlich wie räumlich hinaus auf eine sich fortlaufend reproduzierende Struktur. Butler verweist hier auf die „heterosexuelle Matrix“, bei West \& Zimmerman geht es um die „Omnirelevanz“ der „Sex-Kategorie“ (Kelan 2010), d. h. wir befinden uns fortlaufend in einer Zwangslage, das Geschlecht mit Referenz an die vorgegebenen Sex-Kategorien auch hervorzubringen. Es kann gerade nicht frei nach Gusto „gewählt" werden. Strukturierend - und damit die Situation gestaltend - wirken hier diskursive und körperliche Praktiken, aber auch materielle Artefakte wie z. B. Kleidung, Accessoires oder Werkzeuge, die Goffman (1977/1994) als gender display beschrieben hat, aber insbesondere auch die ,institutionellen Arrangements" (Goffman 1977/1994), wie z. B. der nach Geschlechter differenzierende Arbeitsmarkt, die Existenz traditioneller Frauen- und Männerberufe, Lohnunterschiede, die Berufswahl, die geschlechterdifferenzierende Arbeitsteilung im Allgemeinen, aber auch im Speziellen am Arbeitsplatz, die Raumordnung und vieles mehr.

Die Strukturen und Normen, auf die die Herstellung von Geschlechterdifferenz situativ jeweils verweisen muss, sind jedoch zunehmend als nicht mehr so klar geschlechterdifferenzierend und „traditionell“ zu beschreiben, wie dies in Goffman und Garfinkels ersten Studien in den 60er Jahren in den USA der Fall gewesen ist (Hirschauer 1994). So konstatieren Beck, Bonß und Lau (2001, S. 23) eine einsetzende „Geschlechterrevolution“. Strittig ist allerdings, inwieweit es sich hierbei um eine „Revolution“ und auch Veränderungen auf institutioneller Ebene handelt und nicht um eine ,rhetorische Modernisierung“ (Wetterer 2003), d. h. ein Streben nach Gleichheit, das sich aber nicht in gleicher Weise in den Alltagspraktiken niederschlägt. Mit der Diskussion der „De-Institutionalisierungsthese“ 
Heintz und Nadais (1998, S. 83) zeigt sich jedoch deutlich, dass sich Geschlecht in der „reflexiven Moderne“ (Beck et al. 2001) „vermehrt über gezieltes Handeln erzeugt und symbolisch markiert werden" muss und weniger über formale Zugangsregelungen z. B. zu Bildung oder rechtliche Ungleichheit stabilisiert werden kann. Die Unterscheidung nach Geschlecht scheint daher weniger stabil und ist stärker von den spezifischen Kontextbedingungen abhängig (Heintz und Nadai 1998, S. 78; Heintz 2001; Wetterer 2006). Dem doing gender ist die Eindeutigkeit abhandengekommen, es ist begründungspflichtig geworden, was jedoch vielmehr auf eine Veränderung der Konstruktionsprozesse und nicht auf einen Bedeutungsverlust der Kategorie selbst verweist (Gildemeister und Robert 2008, S. 232 Fussnote).

Um die Auswirkungen der beobachteten gesellschaftlichen Veränderungen für die soziale Konstruktion von Geschlecht konzeptionell erfassen zu können wurde es notwendig, neben dem doing gender, also der fortlaufenden Herstellung von Geschlecht, auch die Möglichkeit eines undoing gender vorzusehen (Aumais 2017; Butler 2004/2009; Deutsch 2007; Hirschauer 1994; 2001). Undoing gender zu untersuchen bedeutet damit ganz allgemein, nicht nur die fortwährende Wiederholung der immer gleichen Differenzierungs- und Hierarchisierungspraktiken zu untersuchen, sondern ebenso zu analysieren, wo diese im Hintergrund verbleiben (Faulstich-Wieland et al. 2004; Goffman 1977/1994; Hirschauer 1994, 2001) oder gar vergessen werden (Hirschauer 2001), Differenz negieren oder Gleichheit betonen (Deutsch 2007) oder aber die Unterscheidung als solche subversiv unterwandert wird (Butler 2004/2009).

Doing gender als sozialwissenschaftliches Konzept kann dabei auf unterschiedliche „Reisen“ mit auch unterschiedlicher Routenführung in den jeweiligen disziplinären Kontexten (Soziologie, Organisationsforschung, Psychologie, Philosophie) wie auch Sprachräumen zurückblicken (West und Zimmerman 2009). Als travelling concept (Czarniawska und Sevón 2005) hat es in den unterschiedlichen Rezeptionsräumen auch unterschiedliche Konnotationen erhalten, durch die verschiedene Theoriebestandteile mit aufgenommen und weitergetragen, andere aber nicht zwingend aufgegriffen wurden (West und Zimmerman 2009). Doing gender ist dabei zu einem wichtigen Schlüsselbegriff jeglicher sozialwissenschaftlichen Forschung zu Geschlecht geworden, der häufig jedoch im Sinne eines ceremonial citings (Wickes und Emmison 2007) lediglich als allgemeines Bekenntnis zu einem mehr oder weniger sozialkonstruktivistischen Zugang zu Geschlecht verwendet wird. Indem wir doing und undoing gender als travelling concept verstehen, geht es uns an dieser Stelle jedoch nicht darum, eine „richtige“ oder „falsche“ Verwendung der Konzepte zu identifizieren (Risman 2009). Vielmehr möchten wir in diesem Kapitel die verschiedenen Diskussionen eines undoing, 
die in den letzten Jahren an unterschiedlichen Orten stattgefunden haben, genauer darstellen (Aumais 2017; Deutsch 2007; Hirschauer 1994, 2001; Kelan 2010; Nentwich und Kelan 2014; Risman 2009; West und Zimmerman 1987, 2009), die jeweils unterschiedlichen Konzeptionalisierungen herausarbeiten und auf ihre empirische Untersuchbarkeit hin überprüfen.

Was genau ist gemeint, wenn wir von doing und undoing gender sprechen? Geht es im ersten Fall um die fortlaufende Herstellung einer natürlich anmutenden Unterscheidung, so muss deren Herstellung im Falle eines undoing zunichte gemacht oder aber unterlassen werden. Mit der Diskussion von undoing gender rückt das Unterlassen, Hinterfragen, Unterlaufen, schlicht das Verändern des Konstruktionsprozesses in den Mittelpunkt. Wie verändern sich Zuschreibungsprozesse, Inhalte von Geschlechterdifferenz oder aber die Differenzierungspraktiken selbst? Handelt es sich dabei um eine in manchen Kontexten zu diagnostizierende „Neutralisierung“ der zur Verfügung stehenden Infrastrukturen, also einen institutionellen Wandel? Geht doing gender quasi die Infrastruktur aus, wie Hirschauer (2001 S. 231) feststellt? Oder geht es um eine Veränderung der Positionierungspraktiken von Individuen und damit um Veränderungen der Geschlechtsidentität? Stellen wir uns zum Beispiel eine Frau in Führungsposition vor, die nachweislich doing masculinity machen muss, um kompetent zu wirken (Aumais 2017; Risman 2009). Handelt es sich hierbei um ein doing gender, da sie die in diesem Feld verankerte Norm der Führungskraft als männlich inszeniert? Oder aber um ein undoing gender, da ihr doing Führung ihre Weiblichkeit infrage stellt? Was aber genau bedeutet in diesem Zusammenhang weiblich? Allein dieses Beispiel zeigt uns, dass es konzeptionell nicht ganz einfach werden wird.

Dabei ist ein undoing gender leichter zu identifizieren, wenn es sich um das doing gender eines Individuums in einer klar an Zweigeschlechtlichkeit ausgerichteten Gesellschaft handelt. So der Startpunkt Garfinkels (1967). Geschlechtsidentität wird hier artikuliert und steht im Mittelpunkt der Analyse seiner Studien zu Transsexualität. Was aber, wenn wir andere Orte anschauen, in denen Geschlecht nicht das zentrale Thema darstellt, sondern lediglich ein Thema unter vielen möglichen ist (Fenstermaker und West 2002)? Oder wir Gesellschaften anschauen, in denen Geschlecht keine so eindeutige „Platzanweiserfunktion“ mehr übernimmt, wie dies in den USA der 1960er Jahre gewesen ist, sondern sich sogar gesamthaft der Gleichheit verschrieben haben (König 2012)? Was, wenn das doing gender in einer Organisation wie einer Kita stattfindet, die von sich sagt, alle Kinder gleich zu behandeln und das „Kind als Individuum“ in den Mittelpunkt rückt (McNaughton 2000)? Hat hier ein institutionelles undoing stattgefunden, welches sich hier bereits auf die Identitätskonstruktionen der Mitarbeitenden und Kinder auswirkt und zu einem degendering (Lorber 2000) der Institution beiträgt? Diese 
Ausführungen machen deutlich: über undoing gender näher nachzudenken ist ein komplexes Unterfangen!

Undoing gender stellt uns zudem auch vor vielfältige empirische Herausforderungen. Um ein Phänomen empirisch untersuchen zu können, brauchen wir hinreichende Definitionen und Operationalisierungen, um auch bestimmen zu können, wann und wie wir es identifizieren und interpretieren können. Nentwich und Kelan (2014) kritisieren hier die empirische Umsetzung vieler doing gender-Studien als zu wenig präzise hinsichtlich des Untersuchungsgegenstands, wie auch der untersuchten Phänomene und ihrer Konzeptionalisierungen. Unter dem Deckmantel von doing gender wird zwar jeweils angegeben, den Prozess der Herstellung von Geschlecht zu untersuchen. Was jeweils im Einzelnen hergestellt wird (Sex-Kategorisierung, Identität oder Hierarchie; Männlichkeit, Weiblichkeit oder aber etwas anderes) ist dabei jedoch sehr heterogen. Als problematisch gilt auch, dass die Annahme der sozialen Konstruktion von Geschlecht häufig unter der Annahme klar definierter Verständnisse von ,Weiblichkeit“ und „Männlichkeit" erfolgt, wenn zum Beispiel ohne weitere Erläuterung soft managerial practices als „,weiblich“ und teamwork als „männlich“ analysiert wird (Fournier und Smith 2006). Eine entscheidende Herausforderung für die empirische Forschung besteht insbesondere darin, analytisch nicht die binäre Unterscheidung einzuführen, deren Mechanismen eigentlich untersucht werden sollen - Zweigeschlechtlichkeit also nicht zu ,reifizieren“, d. h. zu vergegenständlichen (Berger und Luckmann 1966/1996, S. 95 ff.). Um dem Anspruch einer ,reflexiven Geschlechterforschung“ - d. h. empirische Forschung, die in systematischer und reflektierter Weise mit der Gefahr der Reifikation umzugehen vermag (Kelle 2016, S. 7-8) - nachzukommen, sollte Geschlechterdifferenz nicht angenommen, sondern empirisch auf ihre Herstellungs- und Wirkungsweisen untersucht werden. Wenn wir uns in den folgenden Abschnitten nun mit verschiedenen konzeptionellen Überlegungen zu undoing gender beschäftigen, dann immer auch hinsichtlich der Frage, wie ein bestimmtes Konzept empirisch umgesetzt werden kann und dabei erlaubt, sowohl doing als auch undoing gender zu untersuchen, ohne es zugleich unkritisch zu reifizieren.

\subsubsection{Von der „Omnirelevanz" zur „Omnipräsenz": (Un)doing gender als kontextabhängig und episodenhaft}

Könnten wir einfach damit aufhören, Geschlecht fortlaufend herzustellen? Diese Frage stellten sich die Ethnomethodologen West und Zimmerman bereits in ihrem 1987 veröffentlichten Artikel und beantworten sie mit einem klaren „nein“ 
(S. 137). Dies, da unter doing gender im ethnomethodologischen Verständnis Geschlecht als die grundsätzliche Kategorisierbarkeit jeglichen Verhaltens entlang der zweigeschlechtlichen Norm verstanden wird. Mit dem Begriff der accountability fassen West und Zimmerman (1987; Hollander 2013) die Wirkung von Institutionen und Normen und verorten sie in der Interaktion. Wir müssen uns zu erkennen geben, da wir für unser Geschlecht accountable, d. h. verpflichtet sind, uns als kategorisierbar erkennen zu geben. Dabei ist jedoch unerheblich, ob das, was getan wird, der Norm entspricht, d. h. einer bestimmten normativen Vorstellung von ,richtiger“ Männlichkeit oder Weiblichkeit. Wichtig ist, dass alle kategorisierbar sein müssen: „managing the occasions so that, whatever the particulars, the outcome is seen as gender-appropriate or, as the case may be, gender-inappropriate, that is, accountable" (West und Zimmerman 1987, S. 135). Omnirelevanz der Geschlechtskategorie bedeutet folglich, dass die „SexKategorie" als Eintrittskarte jeglicher sozialer Interaktion verlangt wird, und die Interagierenden auch zur Rechenschaft gezogen werden würden, würden sie sich nicht erkennbar machen. „Die Hervorbringung von Geschlecht, Ethnie, Rasse bedeutet, dass man das Handeln an den normativen Vorstellungen darüber ausrichtet, wie sich ein Mitglied einer bestimmten Ethnie, Klasse oder Geschlechtskategorie angemessen zu präsentieren und zu verhalten hat." (Fenstermaker und West 2001, S. 244) Undoing findet in diesem Verständnis demnach nur dann statt, wenn Individuen nicht mehr entlang der Sex-Kategorie kategorisiert werden würden und in diesem Moment nicht mehr accountable wären, wie West und Zimmermans Auseinandersetzung (2009) insbesondere mit Risman (2009) deutlich zeigt.

West und Zimmerman (2009) verstehen doing gender konsequent als auf die Sex-Kategorisierung bezogen, die von Deutsch (2007) und auch Risman (2009) thematisierten Veränderungen auf normativer Ebene, nämlich was unter gender verstanden werden kann, liegen nicht unmittelbar in ihrem Fokus. Empirisch ist dies relevant, da hier konsequent von der Interaktion her gedacht wird: doing gender ist immer das, was in einer bestimmten Interaktion hergestellt wird, um eine eindeutige Sex-Kategorisierung hervorzubringen. Die von Deutsch (2007) und auch Risman (2009) thematisierten Aspekte, dass Individuen sich entlang eines anderen Verständnisses von Männlichkeit oder Weiblichkeit verhalten oder aber die Kategorisierung selbst infrage stellen, sich also widerständig verhalten, können demnach in diesem ethnomethodologischen Verständnis nicht als undoing gender konzeptionalisiert werden. 
Undoing gender wird bei West und Zimmerman verstanden als eine Aufhebung der accountability hinsichtlich des Geschlechts. Undoing wäre gleichbedeutend mit der Aufhebung des Zwangs, nach Sex-Kategorie identifizierbar zu sein.

Die von West und Zimmerman gesetzte „Omnirelevanz“ ist vor diesem Hintergrund zu verstehen - es ist keine Interaktion denkbar, ohne dass die daran Beteiligten sich nicht entlang der Sex-Kategorie zu erkennen geben müssen. Die möglichen Inhalte, mit denen „Männlichkeit“ und „Weiblichkeit“ identifiziert werden könnten, können nur durch ihren Effekt auf die Sex-Kategorisierung benannt werden, sie sind keinesfalls als ,fixed set of specifications“ (West und Zimmerman 2009, S. 117) zu verstehen.

Begrifflich führen Gildemeister und Hericks (2012, S. 302 ff.) die Omnipräsenz als Alternative zur Omnirelevanz ein. Geschlecht sei zwar allgegenwärtig, jedoch nicht unweigerlich auch in jeder Situation relevant. Sie betonen die „kontextuelle Kontingenz", das heisst Geschlecht wird in bestimmten Situationen relevant gemacht, in anderen wiederum verbleibt es im Hintergrund und wird nicht zum „Thema“" gemacht. Mit diesem Verständnis verweisen sie auf gesellschaftlichen Veränderungen, durch die die Geschlechterklassifikation ihre Eindeutigkeit verloren habe. Auch Thorne (1993), Hirschauer (1994) und Deutsch (2007) betonen die Relevanz des Kontexts. Hirschauer (1994) weist dabei darauf hin, dass die Omnirelevanzannahme insbesondere aus den Studien an transsexuellen Menschen abgeleitet wurde. Während er die Omnirelevanz des Geschlechts für die Alltagserfahrungen von transsexuellen Menschen für zutreffend hält, so sei doch fraglich, ob hiervon in anderen sozialen Situationen auch auszugehen sei. Deutsch (2007, S. 108) fragt, inwieweit Geschlecht in unterschiedlichen Gesellschaften zu unterschiedlichen Zeitpunkten und auch an unterschiedlichen Orten innerhalb einer Gesellschaft auf unterschiedliche Weise relevant gemacht wird: „Gender is produced differently among white blue-collar laborers, unemployed African Americans, white software developers, and Black physicians." Kurzum: Geschlecht wird kontextualisiert hervorgebracht und die Relevanz von gender für eine bestimmte Interaktion wird hier nicht mehr per se angenommen, sondern zur zentralen zu untersuchenden empirischen Frage gemacht. Mit der Frage nach dem undoing gender wird der Anspruch an empirische Forschung gestellt, doing gender Prozesse mit einer grösseren Offenheit auf Seite der Forschenden zu untersuchen. Es könnte nicht nur das bereits Bekannte, sondern auch etwas ganz Anderes relevant gemacht werden. Empirische Forschung zu (un)doing gender sollte somit nicht 
bei der Suche nach Geschlechtsunterschieden stehen bleiben (Hirschauer 2014, S. 183 Fussnote).

Den episodischen Charakter der Herstellung von Geschlecht verdeutlichend verweisen Deutsch (2007) und auch Hirschauer (1994) auf das sozialpsychologisch gut beschriebene Phänomen der „Salienz“ (Taylor und Fiske 1978). Zwar ist die Sex-Kategorie allgegenwärtig und potenziell in jeder Situation verfügbar - omnipräsent - jedoch zeigen vielfältige Studien auf, dass sie auch im Hintergrund ,ruhen“ gelassen werden kann. Geschlecht kann salient sein, d. h. aktiviert werden, dies muss aber nicht erfolgen (Ridgeway und Correll 2004), es kann auch dethematisiert werden (Müller et al. 2007; Pasero 1995). Während die Sex-Kategorisierung in der Tat fortlaufend erfolge, Hirschauer (2012, S. 160) spricht hier im Sinne der accountability von einem durchaus bestehenden „Ausweiszwang", kann gender in manchen Momenten in den Mittelpunkt gerückt, in anderen aber auch im Hintergrund bleiben und ,ruhen“. Hirschauer und auch Deutsch betonen den episodischen Charakter der Herstellung von Geschlecht. So finde zu Beginn einer Interaktion, u. a. angeregt durch die häufige visuelle Präsenz des gender displays (Goffman 1977/1994), ein gegenseitiges Klassifizieren statt. Die empirisch interessante Frage ist jedoch, ob diese ,initiale Geschlechtsunterscheidung im Verlauf der Interaktion" (Hirschauer 2012, S. 160) auch aktualisiert, oder aber neutralisiert werde (Hirschauer 2001, S. 217). Im Vokabular der Ethnomethodologie wird die Sex-Kategorie zugewiesen, das daran anschliessende doing gender muss jedoch nicht zwingend stattfinden. Hirschauer fragt, inwieweit die Interaktionsteilnehmenden, sind sie einmal als Männer und Frauen klassifiziert, auch in ihrer Geschlechtlichkeit adressiert werden. Dies kann zum Beispiel durch direktes Ansprechen einer Person als Frau oder als Mann geschehen. Neben einer solchen deutlichen Adressierung des Geschlechts ist es aber auch möglich, Geschlecht zu dethematisieren, es beiläufig zu tun, Geschlechterunterscheidungen herunterzuspielen oder zu konterkarieren - Hirschauer (2001, S. 218) beschreibt dies als unterschiedliche Aktivierungsgrade der Adressierung. Doing gender findet in Hirschauers (2001, 2016, S. 117) Verständnis dann statt, wenn Geschlecht direkt adressiert wird, ein undoing, wenn dies unterbleibt oder konterkariert wird oder aber gar ganz „vergessen“ wird.

Deutsch und Hirschauer verstehen unter undoing gender, dass Geschlecht in der Interaktion nicht salient gemacht wird. Es kann im Hintergrund verbleiben, gänzlich irrelevant werden oder vergessen werden (not doing gender). 
Deutsch (2007) gründet ihr Verständnis von undoing stärker in der sozialpsychologischen Forschung und insbesondere ihren begründeten Zweifel an der kognitionspsychologischen These der automatischen Aktivierung der Geschlechtskategorie in jeglicher Interaktion (Ridgeway und Corell 2004). Am Beispiel einer Chinesin führt Deutsch aus, dass Geschlechtsidentität dann salient werden kann, wenn wir die Frau beim Auflegen von Make-up beobachten, jedoch eher ihre chinesische Identität in den Vordergrund tritt, beobachten wir sie beim Essen mit Essstäbchen. Es ist die Lenkung unserer Aufmerksamkeit in der beobachteten Situation, die nach Deutsch Einfluss auf die Salienz einer bestimmten Identitätskategorie hat und dazu führen kann, dass gender unter bestimmten Bedingungen sogar so irrelevant werden kann, dass wir es nicht erfassen oder bemerken (Deutsch 2007, S. 116). Irrelevanz bedeutet hier, dass eine andere Facette der Identität Aufmerksamkeit erhält. Auch bei Hirschauer steht Geschlecht in Konkurrenz zu anderen möglichen „Mitgliedschaften“ (2016). Mit der Möglichkeit, das „Vergessen des Geschlechts“ (2001) zu denken beschreibt er ein „Indifferenzverhältnis, das Praktiken und Strukturen entkoppelt, sodass Geschlecht schlicht nicht stattfindet." Hirschauer (2016, S. 218) unterscheidet damit schlüssig das undoing gender, das im binären System der Geschlechterdifferenz verbleibt vom ,not doing gender, but anything else" als die Praktiken, ,,in denen Geschlecht nur noch in Spurenelementen oder überhaupt nicht mehr sinnhaft enthalten ist".

Wie aber können wir empirisch ein doing vom undoing oder gar not doing unterscheiden? Welches Tun, welche Symbole, welche Gesten sollen als doing und welche als undoing gender interpretiert werden? Deutsch schlägt vor, den Begriff des doing gender für Interaktionen zu verwenden, in denen Geschlechterdifferenz produziert wird, während undoing gender für Interaktionen reserviert bleibt, in denen Differenz reduziert bzw. Gleichheit betont wird (Deutsch 2007, S. 122). Die Definition von doing und undoing gender nimmt so die jeweilige Erzeugung oder Ablehnung von Geschlechterdifferenz auf und ist zugleich konsequent empirisch angelegt. In ähnlicher Weise schlägt Hirschauer (2014, S. 183) vor, für das undoing die in der Interaktion vorhandenen Erwartungsstrukturen und Normalitätsannahmen sowie nicht stattfindende Adressierungen und nicht aktualisierte Klassifikationen als empirische Marker zu nutzen (Hirschauer 1994, S. 678 ff., 2001, S. 220 Fussnote). Enttäuschte Erwartungen machen offensichtlich, was unterlassen wurde. Eine Handlung könnte dann als undoing interpretiert werden, wenn sie eine Unterscheidung zurückweist, unterbindet oder verhindert (Hirschauer 2001, S. 214 ff.). 
Deutsch versteht unter undoing gender Interaktionen, in denen Geschlechterdifferenz reduziert bzw. Gleichheit betont wird, im Unterschied zu Praktiken des doing gender, durch die Geschlechterdifferenz produziert wird.

Hirschauer (2016) kritisiert jedoch an Deutschs Definition des undoing gender, dass im Rahmen einer binär-zweigeschlechtlichen Logik verblieben wird - eine Unterscheidung nach Geschlecht wird hergestellt, oder aber eben nicht. Verbleibe die Analyse innerhalb dieser binären Logik, so Hirschauer, könne das „Vergessen“ von Geschlecht - den Fall, den er als ,not doing gender“ bezeichnet - nicht vollständig analysiert werden. Das „Vergessen“ des Geschlechts verweist demnach auf eine Interpretation, die ausserhalb des binären Rahmens von Differenz und Gleichheit liegen muss. Wie aber können wir erkennen, dass es sich bei einer Interaktion um doing gender, einer anderen um undoing gender, in einer dritten dann aber um etwas ganz anderes handelt, das ausserhalb dieses binären Rahmens liegt? Wie lässt sich ein not doing gender identifizieren, das eben gerade nicht passiert? Die methodische Herausforderung, not doing zu untersuchen ist der dabei vorliegende negatorische Charakter. Hirschauer (2014, S. 183) schlägt wiederum einen analytischen Zugang über die enttäuschten Erwartungsstrukturen oder Normalitätsannahmen vor: Ein not doing oder die von Deutsch thematisierte Irrelevanz von Geschlecht kann nur dann analysiert werden, wenn offenkundig etwas anderes als das von der Beobachterin Erwartete ins Zentrum der Aufmerksamkeit rückt (Hirschauer 2014, S. 183).

Hirschauer (2016, S. 118) unterscheidet undoing gender als eine „negatorische Aktivität im Horizont der Geschlechterdifferenz" vom not doing gender als den Praktiken, ,,in denen Geschlecht nur noch in Spurenelementen oder überhaupt nicht mehr sinnhaft enthalten ist“.

Während im ethnomethodologischen Verständnis ausschliesslich die Relevanzsetzungen, Adressierungen und Aktivierungen der Interaktionsteilnehmenden als Grundlage einer legitimen Interpretation angesehen werden, werden von Hirschauer auch die Forschenden zu legitimen Interpretinnen und Interpreten des beobachteten (un)doing gender.

Inwieweit und unter welchen Vorzeichen es nicht nur erlaubt, sondern geradezu notwendig ist, über das in der Situation vorhandene hinaus Bezüge herzustellen, 
wurde ausführlich in der Auseinandersetzung zwischen dem Konversationsanalytiker Schegloff (1997), der Sozialpsychologin Wetherell (1998) sowie dem Sozialpsychologen Billig (1999a, 1999b) diskutiert. Wetherell und Billig argumentieren diskurstheoretisch und konstatieren, dass Verstehen nur jeweils innerhalb eines kulturell gewachsenen Bedeutungsrahmens möglich ist. Zwar wird die Relevanz der ethnomethodologischen Forderung, dass jegliche Interpretation im analysierten Material begründbar sein müsse (Lynch 2001), als ein wichtiges Gütekriterium der empirischen Datenanalyse betont (Wetherell 1998). Zugleich wird aber aufgezeigt, dass Interpretationen ohne Bezugnahme auf sogenannte „Proto-Konversationen“ (Shapiro 1992) schlicht unmöglich sind. Es handelt sich dabei um die historischen Entwicklungen der Elemente des Gesprächs, die dessen Durchführung jeweils erst ermöglichen. Diese „Proto-Konversationen“ müssen jeweils identifiziert werden. Eine Interaktion rein aus Sicht der Teilnehmenden verstehen zu wollen, wie vom Konversationsanalytiker Schegloff (1997) gefordert, ist aus dieser Perspektive ein unmögliches, ja sogar naives Unterfangen (Billig 1999b), geht es doch davon aus, Wirklichkeit könne beobachtet und ,aufgezeichnet“" werden.

Billig (1999a) zeigt die Unmöglichkeit dieses Unterfangens ausführlich anhand verschiedener Transkriptionskonventionen auf. Werden die Sprechenden mit „speaker A“ und ,speaker B“ bezeichnet, so geht dies auf eine zumindest implizit getroffene Entscheidung der Forschenden zurück, die Sprechenden als geschlechtslos und gleich gestellt darzustellen. Werden sie hingegen - wie im von Schegloff (1997) verwendeten Beispiel geschehen - mit Vornamen versehen, sind sie klar nach Geschlecht kategorisierbar. Geschlecht ist qua Transkriptionskonvention in der interpretierten Szene enthalten, auch wenn die Teilnehmenden sich nicht explizit darauf beziehen. In dieser Auseinandersetzung wird deutlich, dass es sich bei einer Analyse von doing und undoing gender, die sich ausschliesslich auf die Aktualisierungen in der beobachteten Interaktion bezieht, um eine gravierende Engführung handelt (Billig 1999a, 1999b). Insbesondere die durch die Interaktionen hervorgebrachten Institutionalisierungsprozesse und ihre Relevanz für das doing und undoing gender können so nicht untersucht werden, da Institutionen im ethnomethodologischen Verständnis lediglich als Ressourcen den Interaktionsprozessen zugrunde liegen (Gildemeister 2017, S. 4; Hirschauer 2016). Einen Ausweg aus diesem Dilemma sieht Wetherell (1998) in einer stärker sozialtheoretischen Fundierung des (un)doing gender Verständnisses (Wetherell 1998) und insbesondere einer grösseren Relevanz des Institutionenbegriffs (Gildemeister 2017; Hirschauer 1994). Ein deutlicheres Verständnis des Zusammenspiels von Identität, Geschlecht und kulturell tradierten und damit institutionalisierten Wissensbeständen bzw. den „Infrastrukturen der Geschlechter(in)differenz“ 
(Hirschauer 2012) wird notwendig, um die mit der empirischen Suche nach undoing gender verbundenen Unsicherheiten und Vagheiten zu überwinden.

Wir wenden uns in den nächsten Abschnitten zunächst Goffman und insbesondere seinem Begriff der ,institutionellen Reflexivität“ zu, mit dem ein über die Situation hinausreichendes Verständnis der kulturellen und institutionellen Faktoren entwickelt wird (Hirschauer 1994). Mit dem Begriff der „Dramatisierung“ (Faulstich-Wieland et al. 2004) kann zudem der episodische Charakter des doing und undoing gender näher beschrieben werden. Es folgt eine Auseinandersetzung mit dem Butlerschen Performanzbegriff, durch den die Goffmanschen Ausführungen nochmals einen neuen Zuschnitt erhalten, gefolgt von beide Ansätze weiterführenden praxistheoretischen Überlegungen.

\subsection{2 "Institutionelle Reflexiviät" und „Genderismen“: (Un)doing gender als Dramatisierung und Entdramatisierung}

Während der institutionelle Rahmen aus ethnomethodologischer Sicht lediglich die Ressource für Interaktionsprozesse bereit stellt, wird er dort nicht selbst zum Forschungsgegenstand gemacht (Gildemeister 2017, S. 412), ein Umstand, den Goffman schon früh kritisiert hat. Mit seinem 1977/1994 erschienen Aufsatz „The Arrangement between the Sexes“ entwickelt er ein Verständnis der ,institutionellen Reflexivität“. Er bezeichnet hiermit die ,andernorts fabrizierte(n) 'Halbfertigteile" " (Hirschauer 1994, S. 680), d. h. die der beobachteten interaktiven Geschlechterdarstellung zur Verfügung stehende Infrastruktur. Hierzu gehören z. B. das gesellschaftlich etablierte Wissen über Zweigeschlechtlichkeit, die individuellen Biografien als geschlechtliche Wesen, die semiotische Stabilität des Verweisungszusammenhangs von Zeichen sowie die sozialstrukturelle Stabilität der institutionellen Arrangements der Geschlechterbeziehungen (Goffman 1977/1994, S. 107 ff.; vgl. Hirschauer 1994, S. 680 ff.; Hirschauer 2012). Diese Infrastrukturen haben über die eigentliche Situation der Interaktion hinweg Bestand und erzeugen die Stabilität des zweigeschlechtlichen Kategorisierungssystems. Sie können jeweils situativ bestätigt werden, wodurch wiederum die institutionellen Arrangements legitimiert werden. Hierin sieht Goffman die Reflexivität begründet. Beispiele für solche institutionellen Genderismen sind bei Goffman heterosexuelle Paarbeziehungen, die Arbeitsteilung und auch die geschlechterdifferenzierende Zuschreibung von Berufen, wie auch die Trennung der Toiletten im öffentlichen Raum und andere, in die materielle Ordnung eingeschriebene Unterscheidungen. So zeigen verschieden Studien auf, wie z. B. 
die Sitzordnung im Schulalltag einen Genderismus darstellt und für die Darstellung von Geschlecht genutzt werden kann (Faulstich-Wieland 2000, zitiert in Faulstich-Wieland 2001, S. 7 Fussnote).

Während diese institutionellen Arrangements die Natürlichkeit eines Geschlechtsunterschieds inszenieren, müssen sie zugleich nicht zwingend in jeder Interaktion Verwendung finden. Faulstich-Wieland et al. (2004, S. 23) betonen aufbauend auf Goffmans (1959/1976) dramaturgischen Begrifflichkeiten des „Selbst und seinen Inszenierungen“ die Relevanz der situativen „Darstellung" (performance im englischen Original) von doing gender: Geschlecht kann demnach „dramatisiert“ werden und damit ins Zentrum der Interaktion rücken, oder aber „entdramatisiert“ werden und im Hintergrund verbleiben bzw. keine Rolle mehr spielen. „Die Frage, ob eine soziale Situation ein Schauplatz für Geschlechtskonstruktion ist oder nicht, wird von den Teilnehmenden ausgehandelt. Institutionelle Trennungen (wie Damen- und Herrentoiletten oder auch wie geschlechtshomogene Gruppen in Schulen) aktualisieren die Geschlechterdifferenz nach aussen und können - müssen allerdings nicht - sie nach innen neutralisieren" (Faulstich-Wieland et al. 2004, S. 24). Dies ist zum Beispiel dann der Fall, wenn ein durch gender display eingebrachter Aspekt (z. B. ein Aufdruck auf dem T-Shirt) in der Interaktion nicht aufgegriffen wird. Wird die Symbolik jedoch in der Interaktion aufgegriffen, so erfolgt dies häufig mit einer affektiven Besetzung, d. h. es wird Begeisterung oder Missfallen bezüglich der Symbolik geäussert. Dramatisiert werden kann durch sprachliche aber auch nichtsprachliche Praktiken wie Gestik, Mimik, Kleidung, Symbolik, oder das durch Gruppeneinteilung praktizierte borderwork (Thorne 1993), ebenso kann aber auch die einmal hergestellte Relevanz wieder zurückgenommen, entdramatisiert werden.

Bei Goffman ist doing gender als Dramatisierung der institutionell zur Verfügung stehenden Geschlechtsunterscheidungen zu verstehen. Doing gender kann über den Bezug auf stabil zur Verfügung stehende Genderismen identifiziert werden. Faulstich-Wieland, Weber und Willems ergänzen entlang Goffmans Terminologie hierzu die Möglichkeit eines undoing gender, was sie als Entdramatisieren einer hervorgebrachten Unterscheidung verstehen.

Das Goffmansche Vokabular ermöglicht, sowohl die Differenz betonenden Interaktionen, als auch diejenigen zu analysieren, in denen nicht das „typische“ stattfindet (Faulstich-Wieland et al. 2004). Indem auf diese Weise auch analysiert 
werden kann, wann Geschlecht im Mittelpunkt, wann im Hintergrund einer Interaktion steht, kann auch die häufig notwendige "Neutralisierungsarbeit“ sichtbar gemacht werden (Hirschauer 1994). So wird die Weiblichkeit von in Männerberufen arbeitenden Frauen allein aufgrund des in diesem Kontext entstehenden Kontrasts salient gemacht. Tragen sie an ihrem Arbeitsplatz zum Beispiel keinen Schmuck, kann das als ein Versuch interpretiert werden, Weiblichkeit zu neutralisieren. Auch Geschlechtsneutralität wird so zu etwas, das aktiv hergestellt wird. Es handelt sich um eine prekäre und anspruchsvolle soziale Konstruktion, die immer wieder durchkreuzt werden kann. Mit Goffmans Verständnis der ,institutionellen Reflexivität" wird zudem eine klare Verbindung zwischen Interaktion und kulturellem Repertoire aufgezeigt die es erlaubt, situativ Bedeutung zuzuweisen. Auf diese Weise kann in der empirischen Analyse über die interpretierte Interaktion hinausgegangen und weitere, über die Situation hinausreichende Wissensbestände, „Genderismen“, zur Interpretation herangezogen werden.

\subsubsection{Performativität und „gender trouble": (Un)doing als subversiver Akt der Subjektpositionierung}

Butler (1989, 1990/1991) bietet mit ihrem Begriff der „Performativität“ ein den Foucaultschen Begriff von Subjektivierung weiter ausführendes Verständnis des vergeschlechtlichten Subjekts (Davies 2006). Während Goffman in erster Linie auf die Dramaturgie der Geschlechterdarstellung fokussiert, geht es Butler insbesondere um die Effekte dieser Darstellung (Horanyi 2014). Performativität ist somit mehr als die situative Darstellung eines Selbst, Performativität erklärt, wie das Selbst durch seine sprachliche wie körperliche Darstellung hervorgebracht wird (Butler 1988). In deutlicher Abgrenzung zu essentialistischen Vorstellungen des Selbst und einer kohärenten und stabilen Geschlechtsidentität (Miller 2000) wird das Selbst bei Butler (1990/1991) zu einem Effekt des Diskurses, der durch ritualisierte Wiederholungen der zitierten Normen entsteht. Indem Normen fortlaufend zitiert werden, werden Subjekte zu dem, was sie glauben zu sein. Wie Butler (2004/2009) deutlich macht, geht es dabei grundlegend um die Anerkennung als menschliches Subjekt. Dieses kann nur durch die Positionen Anerkennung erlangen, die die geltenden sozialen Normen anbieten und erlauben (Wetherell 2010). Indem diese Normen von anderen akzeptierten Positionen zitiert werden, machen sich Individuen lesbar.

Die performative Hervorbringung von Geschlechtsidentität erfolgt durch die fortlaufende Wiederholung von Handlungen innerhalb dessen, was sozial akzeptiert ist. Mit Bezug auf Foucault, Austin, Derrida und Lacan gelingt es Butler 
somit, Institution und Subjektwerdung miteinander zu denken. Diese in einem bestimmten Kontext herrschenden Diskurse können als „Skripte“ betrachtet werden (Salih 2002), die den sozialen Akteur ,,anrufen“ und dabei vorexistieren und die sozial zulässigen Möglichkeiten von Geschlecht und Sexualität so abgrenzen, dass sie natürlich und Teil einer kohärenten Identität erscheinen. Das Subjekt ist jedoch nicht frei in der Wahl, wie es das geschlechtsspezifische Selbst inszenieren will (Butler 1990/1991). ,"The script‘, if you like, is always already determined within this regulatory frame, and the subject has a limited number of 'costumes' from which to make a constrained choice of gender style" (Salih 2002, S. 63). Mit diesem Verständnis von (un)doing gender thematisiert Butler sowohl die einschränkende, das Subjekt limitierende und unter Umständen auch zerstörende Kraft von Normen (Butler 2009/2004), zugleich aber auch deren fluide Seite, die ein bestimmtes Mass an Handlungsmacht für das Subjekt beinhaltet und die Möglichkeit zu gender trouble bereitstellt, der situativen Unterwanderung der herrschenden Normen (Butler 1990/1991).

Mit doing gender kann mit Butler die limitierende Kraft des Normativen, mit undoing gender die Möglichkeit, den bestehenden Code situativ zu unterwandern und über solche wiederholten performativen Akte zu verändern bezeichnet werden. Die grundlegende Unentschiedenheit des einzelnen Aktes bringen wir mit der Schreibweise (un)doing gender zum Ausdruck.

Auf der einen Seite wird doing gender hier zu einer kulturellen Anforderung, der wir uns nicht verweigern können. Zugleich ist das Subjekt aber auch nicht ohne Handlungsmacht oder Wahlmöglichkeiten, denn normative Anrufungen können jeweils auch zurückgewiesen oder unterwandert werden. Unter Bezug auf Foucault nimmt Butler an, dass das vorliegende normative Skript zwar einschränkt, jedoch nicht vollständig bestimmt, wie Geschlechtsidentität jeweils hervorgebracht werden muss. Ein bestimmtes Mass an Improvisation ist dabei notwendig. Die Möglichkeit zur Veränderung liegt somit genau im Zwang der fortlaufenden Wiederholung begründet. Der Handlungsspielraum des Subjekts lässt sich jeweils in den Abweichungen, seien es unbeabsichtigte Unvollkommenheiten oder bewusste Improvisationen (Van Lenning 2004), lokalisieren. Butler lenkt so unsere Aufmerksamkeit auf die grundlegende Ambivalenz jedes performativen Aktes. Einerseits werden Normen zitiert und wiederholt, zugleich aber auch neu interpretiert und damit die Möglichkeit zur Resignifikation gegeben. Genau in dieser Möglichkeit des Unterlaufens der Norm und des Veränderns sieht sie das 
Potenzial für undoing gender (Butler 2004/2009). Die instabile Natur des Selbst rückt so in den Fokus und öffnet Wege, sowohl die fortlaufende Reproduktion, als auch Veränderung zu analysieren. Doing und undoing gender stehen hier in einem äusserst fluiden Verhältnis, dessen Ambivalenz daher besser mit der Schreibweise (un)doing gender wiedergegeben wird.

Identität wird bei Butler zu einer nie abgeschlossenen Herstellungspraxis (Taylor 2015, S. 9), ein Verständnis, das insbesondere durch die Kritische Diskurspsychologie (Morison und Macleod 2013; Nentwich und Morison 2018; Wetherell 2010) aufgegriffen und konzeptionell präzisiert worden ist. Die Diskurspsychologie liefert dabei ein konsequent empirisches Programm zur Erforschung der Geschlechterperformanz (Kelan 2009). Sie entwickelt einen Subjektbegriff, der das Subjekt gerade nicht in der individuellen Psyche, sondern in psychodiskursiven Praktiken (Wetherell 2008) verortet. Diskurspsychologische Studien haben Positionierungspraktiken in vielfältigen empirischen Settings untersucht, wie z. B. Männlichkeitskonstruktionen jugendlicher und erwachsener Männer (Edley und Wetherell 1997; Wetherell und Edley 1999); Arbeitsteilung in heterosexuellen Familien (Morison und Macleod 2013; Nentwich 2008); Frauen in der IT-Industrie (Kelan 2009) sowie emotionale Belastungen von „Karrierefrauen“ (Chowdhury und Gibson 2019). Mittels der analytischen Klammer der „Subjektpositionierungen" können die interaktiv stattfindenden Positionierungspraktiken vor dem Hintergrund des grösseren diskursiven Kontexts analysiert werden. Typischerweise werden mit einer Analyse der in Alltagssituationen zur Anwendung kommenden ,,interpretativen Repertoires“ (Potter und Wetherell 1987) die diskursiven Ressourcen oder grösseren Diskursstränge identifiziert und in ihrer unmittelbaren Verwendung interpretiert (Wetherell 1998; Wetherell und Edley 1999, 2014). Die Kritische Diskurspsychologie stellt so die analytischen Konzepte zur Verfügung, mit denen die von Wetherell (1998) geforderte Verankerung der Analyse der „Macht der Diskurse“ im empirischen Material, zugleich aber auch mittels Interpretation des kulturell-historisch verfügbaren Wissens erfolgen kann (Nentwich und Morison 2018). Die notwendige Offenheit bezüglich des doing, undoing oder gar not doing wird hier durch ein konsequentes Fokussieren auf die Subjektivierungspraktiken ermöglicht. Normative Erwartungen können zitiert, bestätigt oder verworfen werden und Subjektpositionen somit als konform mit den normativen Anrufungen, oder aber widerständig, subversiv oder ganz andere Themen aufnehmend ausfallen. Allerdings fokussieren diskurspsychologische Analysen bisher nahezu ausschliesslich auf gesprochenen Text, der mittels Interviews erhoben wurde (Nentwich und Morison 2018). Die Entwicklung eines konsequenteren Repertoires an Erhebungsmethoden und Analysemöglichkeiten der Materialität von Diskursen ist somit noch ausstehend (DiMasso und Dixon 
2015; Durrheim 2012), weswegen wir uns nun verschiedenen praxistheoretischen Arbeiten zu doing und undoing zuwenden. Sie greifen sowohl Goffmans dramaturgischen Ansatz als auch Butlers Performanzbegriff auf und entwickeln so Konzeptionalisierungen von gender als diskursiver, sozialer und auch materieller Praktiken.

\subsection{4 (Un)doing gender als diskursive, soziale und materielle Praktiken}

Das performative Verständnis Butlers empirisch weiter ausführend hat die praxistheoretische Forschung die Bedeutung diskursiver, sozialer und materieller Praktiken des doing und undoing gender in den Mittelpunkt gerückt (Aumais 2017; Poggio 2006). Die früheren Überlegungen Connells zu Geschlecht als sozialer Praxis (1987) aufnehmend und weiterführend sind insbesondere die Arbeiten des Teams um die italienische Organisationssoziologin Gherardi (1994, 1995) sowie der amerikanischen Soziologin Martin (2001, 2003, 2004, 2006) als grundlegend anzusehen.

Gherardi (1994, 1995) verknüpft mit einer im symbolischen Interaktionismus verankerten Perspektive auf Organisationskultur die ethnomethodologische, auf Interaktion fokussierende Perspektive West \& Zimmermans ("the gender we do") mit einer Ebene des Wissens ("the gender we think"). Gherardis Verständnis von Wissen als sozialer und diskursiver und in ihren späteren Arbeiten auch materieller Praxis ist als Radikalisierung der wissenssoziologischen Perspektive Berger und Luckmanns zu verstehen (Gherardi 2019). Wesentlicher Bestandteil des Geschlechterwissens bzw. der „symbolischen Ordnung“ ist bei Gherardi (1994, 1995) die binäre Gegensätzlichkeit von Männlichkeit/Weiblichkeit, Identität/Differenz, Natur/Kultur in der sozialen Konstruktion von Geschlecht. Indem sie annimmt, dass sich diese Gegensätzlichkeiten gerade aus feministischer Perspektive in Auflösung befinden, geht sie auch davon aus, dass auch die Grenzen zwischen Männlichkeit und Weiblichkeit beginnen, fluide zu werden (Gherardi 1994, S. 598). Mit ihrer an Goffman angelehnten analytischen Unterscheidung von ceremonial work, zeremoniellem Handeln, und remedial work, dem ,reparierenden“ bzw. die Ordnung wiederherstellenden Handeln (Gherardi 1994), ermöglicht sie, diese Fluidität empirisch näher zu beschreiben. Mit ceremonial work beschreibt sie die Möglichkeit, situativ die symbolische Ordnung zu referenzieren. Dabei kann auf die symbolische Ordnung verwiesen werden, es ist jedoch keinesfalls zwingend erforderlich. Zugleich kann eine Verletzung der symbolischen Ordnung durch remedial work wiederhergestellt werden. Ceremonial 
und remedial work werden so zu empirischen Markern für das Stattfinden von (un)doing gender, denn dort, wo diese Praktiken beobachtet werden können, wird die symbolische Ordnung entweder neu hergestellt und bestätigt, oder aber herausgefordert.

Doing gender involves symbols, using them, playing with them and transforming them; it entails managing the dual presence: shuttling between a symbolic universe coherent with one gender identity and the symbolic realm of the 'other' gender. We do gender through ceremonial work and through remedial work. In the former kind of behaviour we stress the difference between the symbolic universes of gender, in the latter we defer the meanings of gender to situated interactions. (Gherardi 1995, S. 131).

Gherardi versteht unter doing gender das Zitieren der symbolischen Ordnung durch ceremonial work, während remedial work zum Einsatz kommt, wenn ein undoing stattfand, d. h. diese Ordnung herausgefordert wurde.

Zentral für die Analyse des Geschlechterwissens sind für Gherardi die diskursiven Praktiken (2019, S. 125 ff.). Der Sprachgebrauch in einer spezifischen sozialen Situation wird als Aktivität gesehen, durch die koordinierte Handlung (coordinated action) hergestellt wird. Durch institutionalisierte diskursive Praktiken werden institutionalisierte Wissensbestände durch das Zitieren dieses Wissens in Alltagssituationen reproduziert und zugleich bestätigt (Bruni et al. 2005, S. 31). Doing gender ist in dieser Perspektive zugleich in institutionalisierten Diskursen wie auch in interaktiven Praktiken verankert und vorhanden (Bruni et al. 2005, S. 41).

In organizational activity, knowing and doing interweave in practical action and common-sense decision-making. The interactive and discursive practices that constitute organizational action at the same time construct organized action, both because 'the essential reflexivity of accounts' (Garfinkel 1967) is used to create a sense of order in action, and because it reflexively creates the context of action. (Bruni et al. 2005, S. 71).

Während das ethnomethodologische Verständnis von doing gender auf die Interaktion fokussiert und mit dem Begriff der accountability die Wirkung von Institutionen in die Interaktion hineinverlegt (West und Zimmerman 2009), entwickelt die praxistheoretische Perspektive das Goffmansche Verständnis ,institutioneller Reflexivität“" weiter (Gherardi und Poggio 2007; Hirschauer 1994; Nicolini 
2013). Geschlecht und Organisationen werden durch spezifische soziale Praktiken (re-)produziert, die Diskurse, Materialität und Identitäten zusammenführen (Bruni et al. 2005; Poggio 2006) und so als die „Infrastrukturen“ des (un)doing gender verstanden werden (Hirschauer 2012). Dies können Geschlechtsstereotype sein, die das Wissen über tradierte Idealbilder transportieren, Konventionen im Umgang der Geschlechter, die geschlechterdifferenzierende Arbeitsteilung, aber auch „materielle Artefakte“ wie z. B. die Kleidung, oder auch das Design von Alltagsgegenständen wie Kinderwägen und Wickeltischen (Hirschauer 2012, S. 156-157). Da Praktiken als performativ verstanden werden, sind hier sowohl die das institutionalisierte Wissen zitierenden, wiederholenden und damit stabilisierenden Routinen, als auch Improvisationen zu verstehen, die mit diesem Wissen brechen oder es verändern können (Janssens und Steyaert 2019; Nicolini und Monteiro 2017; Reckwitz 2002). Der durch die Einführung des Performanzbegriffs erzeugte epistemologische Bruch führt eine weitere Dimension in der Analyse von doing gender ein: "It considers also 'what this doing does', that is, the effects produced by doing gender while producing society." (Gherardi 2019, p. 71).

Praktiken stellen somit immer auch soziale Ordnung her (Connell 1987). Sie enthalten jeweils mehr als das, was in einer Situation getan werden kann bzw. tatsächlich gemacht wird, da sie zugleich auch die normativen Erwartungen an die Situation enthalten (Zietsma und Lawrence 2010, p. 192). Eine praxistheoretische Analyse untersucht jeweils, wie Praktiken durch die Verbindung von Körpern, Werkzeugen, Artefakten sowie Diskursen hergestellt werden. Diskursive Praktiken werden dabei immer auch als materiell verstanden (Gherardi 2016), denn normative Erwartungen werden nicht nur sprachlich, sondern auch durch körperliche Praktiken, organisationalen Routinen und auch der Ästhetik eines Raums oder Gebäudes relevant gemacht (Tyler und Cohen 2010; Wassermann und Frenkel 2015). In diesem Sinne muss der methodische Zugang ein ethnografischer sein. Praxistheoretische Analysen müssen zudem zwingend ethnografische und diskursanalytische Traditionen zusammenführen (Wrana 2012; Wrana und Langer 2007). Analytisch eine zentrale Rolle nehmen dabei wiederum die Subjektpositionierungspraktiken ein, durch die ein empirisch verankertes Verständnis der kontextuell relevanten Normen, aber auch der hierdurch ermöglichten (oder verunmöglichten) Positionierungen sichtbar gemacht werden kann (Bruni et al. 2005; Gherardi und Poggio 2007; Ott et al. 2012).

Für die empirische Forschung ist die Unterscheidung Martins (2001, 2003, 2004, 2006) in gendered practices, den vergeschlechtlichten Praktiken, und dem practicing gender, der situativen Herstellung von Geschlecht, wesentlich. Vergeschlechtlichte Praktiken stellen die in der sozialen Situation zur Verfügung 
stehenden institutionellen Ressourcen und das Geschlechterwissen zur Verfügung, sie sind die ,geronnene Geschichte“, Idealvorstellung, aber auch das Wissen über bestimmte Tätigkeiten. Dieses Wissen kann mit practicing gender aufgegriffen bzw. „mobilisiert“ (Martin 2001) werden - oder aber auch nicht. Mit dieser konzeptionellen Unterscheidung gelingt es Martin (2003), das situative doing (practicing gender) und die dabei vorgenommene Festschreibung oder Unterwanderung von institutionalisierten Praktiken zu unterscheiden, in die Geschlecht bereits eingeschrieben ist (gendered practice). Dank dieser methodologischen Klammern können sowohl die stabilisierenden als auch die fluiden Eigenschaften von Praktiken untersucht werden (Martin 2003). Über die Analyse der in einem Kontext zur Anwendung kommenden gendered practices können lokal die Wissensbestände analysiert werden, mit denen gender, Männlichkeit und Weiblichkeit hergestellt werden können. Indem eine Analyse des institutionalisierten Wissens konsequent mit einer Analyse der im jeweiligen Kontext bestehenden gendered practices verknüpft wird, kann die Analyse von doing und undoing gender empirisch kontextualisiert und verankert werden. Praxistheoretische Analyse von doing und undoing gender erweitern den diskurspsychologischen Blick auf diskursive Praktiken um soziale und materielle Praktiken, die Subjektpositionen, Wissen und Institutionen erzeugen, stabilisieren, aber auch verändern können (Martin 2004).

\subsubsection{Doing und undoing gender: Von der Theorie zur Empirie}

Mit unserer Reise durch die bisherigen Debatten zu doing und undoing gender haben wir verschiedene theoretisch-konzeptionelle Entwicklungen wie auch methodisch-methodologische Herausforderung aufzeigen können. Die gesellschaftliche Entwicklung hin zu einer grösseren Gleichstellung der Geschlechter wie auch die gestiegene Akzeptanz einer grösseren Vielfalt an geschlechtlichen Rollen, Identitäten, Sexualitäten und Lebensweisen hat zu einer grösseren Vielfalt von doing gender jenseits einer klar binär zugewiesenen Geschlechterordnung geführt. Mit der infolgedessen verworfenen „Omnirelevanz“ und der neuen Annahme der „Omnipräsenz" wurde jedoch auch die klare Verbindung situativer Interaktionen mit einer symbolischen Ordnung binärer und natürlich anmutender Zweigeschlechtlichkeit infrage gestellt. Mit Einzug der „Omnipräsenz“ war es nicht mehr zulässig, von der „Sex -Kategorie“ auf gender zu schliessen und umgekehrt (Kelan 2010); d. h. wir können nicht davon ausgehen, dass Frauen ausschliesslich Weiblichkeit und Männer ausschliesslich Männlichkeit herstellen. In der Folge stellte sich die Frage, was unter solch veränderten normativen Bedingungen noch unter doing, was unter undoing gender verstanden 
und wie das eine vom anderen klar unterschieden werden kann. Die in ethnomethodologischer Tradition geführte Debatte betont mit (un)doing gender die Frage, ob normative Erwartungen an eine Situation bestätigt oder infrage gestellt werden (Risman 2009). So auch der Vorschlag Deutschs, doing als das Betonen oder Herstellen von Ungleichheit, undoing als das Betonen oder Herstellen von Gleichheit zu definieren. Definiert man doing und undoing gender entlang der normativen Erwartungen und ihrer situativen Adressierungen verbleibt die Analyse zweifelsohne in einem binären Verständnis von Geschlecht. Zugleich wurde jedoch deutlich, dass Hirschauers (2001) Vorschlag, auch das ,,Vergessen“ des Geschlechts, also das Ruhenlassen des binären Rahmens zu analysieren zwar konzeptionell konsequent ist, jedoch in der empirischen Umsetzung Fragen aufwerfen muss, da mögliche Bezugspunkte für eine Analyse des gerade nicht stattfindenden fehlen. Dem Verweis auf die Notwendigkeit intersektionaler Analysen von Geschlecht im Zusammenspiel mit anderen Identitätskategorien und der damit verbundenen Analyse des situativen Wechselns zwischen diesen möchten wir zwar nicht widersprechen, er löst das grundsätzliche Problem jedoch nur bedingt. Denn auch hier benötigt eine empirische Analyse Wissen darüber, was als Ausdruck welcher Art von Identitätskategorie gelten kann. Diese Herausforderung spitzt sich nochmals zu, wollen wir wie im vorliegenden Band, doing und undoing gender in einem Kontext untersuchen, in denen die Geschlechtsidentität im Unterschied zu den Analysen Garfinkels oder auch Butlers weder explizit im Mittelpunkt steht, noch anderweitig explizit zum Thema gemacht wird - wir also kaum „natürliche“ Relevanzsetzungen von Geschlecht im untersuchten Kontext erwarten können.

Ein Ausweg, so unser den diskurspsychologischen wie praxistheoretischen Autorinnen folgendes Argument, kann darin bestehen, doing und undoing gender stärker sozialtheoretisch zu fundieren. Goffman, Butler, Gherardi und Martin führen konzeptionelle Begriffe ein, die es ermöglichen, situatives Handeln und institutionell-normativen Rahmen in Verbindung zu bringen. Hierdurch wird es möglich, Geschlechterwissen als symbolische Ordnung vom situativen Zitieren dieser Ordnung soweit zu trennen, so dass Wechsel in der Bezugnahme wie auch das Fallenlassen von Geschlecht als interpretativem Rahmen sichtbar gemacht werden können. Analysen des episodischen doing und undoing gender werden vor diesem performativ-praxistheoretischen Hintergrund möglich, jedoch auch methodisch anspruchsvoller, muss für die untersuchten Interaktionen zwingend der jeweils relevante institutionelle Kontext mit analysiert werden.

Im Forschungsprojekt „,(Un)doing gender in der Kita“ haben wir uns dafür entschieden, die verschiedenen konzeptionelle Ansätze des doing und undoing gender 
nicht als sich gegenseitig ausschliessend, sondern als sich bereichernd einzusetzen. Im folgenden Teil dieses Kapitels zeigen wir auf, inwiefern und hinsichtlich welcher Aspekte Schweizer Kindertagesstätten als gendered organisations (Acker 1990) gelten können.

\section{2 (Un)doing gender im Feld der Kindertagesstätte}

Kitas können als gendered organisations untersucht werden. Das von der USamerikanischen Soziologin Acker (1990) entwickelte Konzept beschreibt die grundlegende Durchdringung von Organisationen mit verschiedenen Annahmen über Geschlecht. So werden die gesellschaftlich-historischen Unterschiede zwischen Frauen und Männern in Bezug auf Lohn, Aufgaben und hierarchischen Positionen in der Organisation abgebildet und führen $\mathrm{zu}$ vergeschlechtlichten „Substrukturen“ wie auch normativen Erwartungen an die zeitliche Verfügbarkeit, die Fähigkeiten und Eigenschaften, sowie den Körper und das Geschlecht eines ideal worker (Kelan 2008). Diese werden in alltäglichen Routinen und Praktiken sowie Interaktionen, dem doing gender, reproduziert und durch Bezug auf eine symbolische Ebene legitimiert. Schlussendlich können innerhalb dieser strukturellen und kulturellen Ordnung wiederum vergeschlechtlichte Subjekte entstehen. Acker (1990) kritisiert mit der gendered organisation die bis anhin in der Organisationssoziologie verbreitete „Vorstellung einer entkörperlichten Arbeitskraft“ wie auch ,die Vorstellung geschlechtsneutraler bürokratischer Organisationsstrukturen.“ (Wilz 2008, S. 509).

Die Kita als gendered organisation (Acker 1990) lässt sich in Hinblick auf die historische Entstehung der Organisation und ihrer Organisationskultur, wie auch in Bezug auf das Geschlechterverhältnis der Kita-Mitarbeitenden untersuchen. Kitas, so die Argumentation, stellen als Organisationen ein besonders interessantes Feld dar, um doing und ungoing gender zu untersuchen. Erstens, da Kitas aus ihrer Entstehungsgeschichte heraus als „Mutterschulen“ und „Kinderbewahranstalten" mit Weiblichkeit verknüpft wurden und zugleich von Anfang an bis heute für die Vereinbarkeit der Berufstätigkeit der Mütter benötigt werden. Zweitens, da die Arbeit in den Kitas im geschichtlichen Rückblick wie auch heutzutage ein Frauenberuf darstellt, was sich im geringen Anteil männlicher Kinderbetreuer, aber auch in den Vorstellungen des ideal workers zeigt. 


\subsubsection{Die historische Entwicklung der Kita: Orientierung an Mütterlichkeit, Bedarf an Vereinbarkeit}

Unser Forschungsprojekt ist der Frage, inwieweit es sich auch bei Kitas um vergeschlechtlichte Organisationen handelt, empirisch nachgegangen. Die historische Entwicklung von Kitas und die Verschränkung ihrer Entstehung mit der geschlechterdifferenzierenden Arbeitsteilung und dem bürgerlichen Geschlechtsmodell des 18. Jahrhunderts sind dabei miteinzubeziehen. Mit der Ablösung der von der Agrarwirtschaft geprägten Grossfamilie der frühen Neuzeit, in denen auf die Mitarbeit von Männern, Frauen wie auch Kindern abgestützt wurde und Kleinkinder oft von weiteren Haushaltsangehörigen betreut wurden, wurde das bürgerliche Familienbild mit einer Trennung von privater Familienund Erziehungsarbeit als Domäne der Ehefrau und Mutter und der öffentlichen Erwerbsarbeit als Aufgabe des Ehemanns und Vaters als Ideal postuliert (Joris und Witzig 1995, 2001; Maihofer 2004; Meuser 2009). Das bürgerliche Familienbild war als Idealbild prägend, auch wenn es nur von bestimmten gesellschaftlichen Schichten realisiert wurde und auf weiteren wirtschaftlichen und gesellschaftlichen Machtverhältnissen wie Dienstpersonal beruhte (Koschorke et al. 2010).

Die Industrialisierung führte dazu, dass ebenso Arbeiterinnen wie Arbeiter für die Fabrikarbeit herangezogen wurden. Frauen ermöglichte die Arbeit in der Industrie eine bessere Arbeit als diejenige einer Dienstmagd (Koschorke et al. 2010). Im Gegensatz zur bürgerlichen Schicht arbeiteten in der Arbeiterschicht auch die Ehefrauen und Mütter in der Fabrik, denn der Lohn des Ehemannes und Vaters alleine reichte für den Unterhalt der Familie nicht aus (Joris und Witzig 1995, 2001). Die ersten Angebote institutioneller Kinderbetreuung wurden für die Kinder dieser Familien aufgebaut. Sie wurden auch „Bewahranstalten“ genannt - die Kinder wurden ,aufbewahrt“, bis die Mutter sie nach der Arbeit wieder betreuen konnte (Roßbach und Grell 2012, S. 333). Die Entstehung der Kita wie auch ihrer Nutzung ist über Dekaden mit der fortschreitenden Industrialisierung verbunden. Noch in den 1960er Jahren waren die Eltern der Kinder, die eine Kita in der Stadt Genf besuchten, mehrheitlich aus der Arbeiterschicht; erst gegen Ende des 20. Jahrhundert zeigt sich eine stärkere soziale Durchmischung in den Kitas (Grob-Menges 2009).

Die Pädagogik des frühen 19. Jahrhunderts beinhaltete Überlegungen zur Entwicklung, zum Lernen und zur Bildung der Kinder in den ersten Lebensjahren. Die neu aufgebauten Kitas orientierten sich dabei an Pestalozzi und Fröbel (Reyer und Franke-Meyer 2016). Pestalozzi und Fröbel betonten, dass die Mutter in den ersten Jahren für Erziehung und Bildung zuständig sein soll. Die Bildung dieser 
ersten Jahre wurde häufig mit Emotionalität, Sozialisation und Werterziehung verbunden, und nicht mit Wissensinhalten und Kompetenzen. Als Konsequenz dieser Betonung der Rolle der Mutter für die frühkindliche Bildung wurde die familienergänzende Betreuung kleiner Kinder im Vergleich mit der Betreuung durch die Mutter zu Hause als weniger wertvoll betrachtet. Die Kindertagesstätten wurden als zweitbeste Lösung für jene Familien vorgeschlagen, für die das Ideal der für Kinderbetreuung und Haushaltführung zuständigen Mutter nicht erreichbar war und für deren Kinder Betreuung dennoch gewährleistet werden musste. Die ersten Kindertagesstätten waren bemüht, einen behüteten und familienähnlichen Ort darzustellen. Wenn Kitas auch nur als zweitbeste Lösung für das Aufwachsen der Kinder galten, sollen sie doch dem Idealbild der Bildung und Betreuung durch die Mutter zu Hause möglichst nahekommen. Höltershinken und Kasüschke (1996, S. 13) beispielsweise fassen die Geschichte der Kinderbetreuung im Ruhrgebiet als Darstellung einer ,, behütete(n) Kindheit“ im Schatten der Fördertürme und Schlote" zusammen. Mit der Gestaltung der Kita als dem familialen Zuhause nachempfunden wird bis heute versucht, nicht gegen den Mythos der ,guten Mutter" (Friis 2008, S. 24) zu verstossen. Eine heimelige Kita, so wird angenommen, entgeht eher der Kritik, dass Kindertagesstätten für die Kinder schädlich seien, da nur die Mutter, natürlicherweise und intuitiv, für ihr Kind am besten sorgen könne.

Kitas wurden in erster Linie gegründet, um Kinder ,,vor , Vernachlässigung und Verwahrlosung' zu schützen", sie nach kirchlichen und gesellschaftlichen Ordnungsvorstellungen zu erziehen, frühes Lernen als Grundlage der ökonomischen Konkurrenzfähigkeit zu sichern, aus christlich-sozialer Wohltätigkeit und auch, „,um den Kindern eine 'kindgemässe und behütete Kindheit' zu gewähren“ (Höltershinken und Kasüschke 1996, S. 13). Diese Motive führten auch dazu, dass die Kitas von Anfang an Kindern jeglichen Geschlechts offenstanden. Wenn die Mütter wegen ihrer Fabrikarbeit die Kleinkinder nicht betreuen konnten, wurden Jungen wie Mädchen von den Kleinkinderzieherinnen in der Kita betreut.

Unterschiedliche Motive und Zielgruppen zeigen sich weiter in den historischen Bezeichnungen und Typen von Kitas (Roßbach und Grell 2012). Mitte des 19. Jahrhunderts gründeten in Deutschland Wirth „Bewahranstalten“ und Fliedner „christliche Kleinkinderschulen“. In diesen stand die Betreuung im Vordergrund, während im „Kindergarten“, wie ihn Fröbel konzipierte, stärker auf die kindorientierte Entwicklung und die Bildung von Fähigkeiten geachtet wurde. Stärker bildungsorientierte Einrichtungen des Typus „Kindergarten“ wurden auch von Kindern aus bürgerlichen Familien genutzt. Die Einrichtungen wurden jedoch differenziert nach Schichtzugehörigkeit ausgerichtet, es gab Einrichtungen für 
Kinder der Arbeiterinnen und solche für die bürgerliche Mittel- und Oberschicht (Reyer 2006).

Wie Roßbach und Grell (2012) argumentieren, zeigen sich diese zwei Traditionen weiterhin: Einerseits das Verständnis in der sozialpädagogischen Tradition, bei dem unter dem Stichwort 'Ganzheitlichkeit' Sozialisation und Betreuung zentral ist (Roßbach und Grell 2012). Andererseits das Verständnis von Kitas und Kindergärten als Vorschule, bei der die Vorbereitung auf die Schule und beispielsweise Vorläuferkompetenzen für den (Schrift-)Spracherwerb im Vordergrund stehen. International zählen Dänemark, Deutschland, Finnland, Norwegen, Österreich und Schweden zur sozialpädagogischen Tradition, Australien, Belgien, Frankreich, Irland, die Niederlande, Großbritannien und die USA (Roßbach und Grell 2012, S. 332) als Beispiele für den Vorschultyp. In der Schweiz zeigen sich diese internationalen Einflüsse in den unterschiedlichen Orientierungen der verschiedenen Sprachregionen. Entsprechend werden Kitas in der deutschsprachigen Schweiz traditionell stärker als Betreuungsinstitutionen verstanden als in der französischsprachigen Schweiz, wo beispielsweise auch heute noch mehr Kinderbetreuende über höhere Berufsabschlüsse verfügen (Müller et al. 2017). Es ist zu vermuten, dass der Unterschied zwischen den Sprachregionen mit diesen international feststellbaren Unterschieden zusammenhängt, dass also die Kita in der deutschsprachigen Schweiz vermehrt als ,Hüte-Einrichtung (Grob-Menges 2009) oder als ,Kinderaufbewahrung ' (Roßbach und Grell 2012) verstanden wird und darum ein weniger hoher Professionalisierungsgrad verlangt wird als in der französischsprachigen Schweiz.

Die absolute Anzahl der Institutionen und Betreuungsplätze hat in den letzten Jahren stark zugenommen, dennoch mangelt es in den meisten Regionen noch immer an Kita-Plätzen (Stern et al. 2018). In der französischsprachigen Schweiz und in den grossen Städten besteht ein grösseres Angebot, in ländlichen Regionen gibt es häufig keine institutionalisierten Angebote (BfS 2019). Zudem sind die Kosten in der Schweiz im Vergleich zu den Nachbarländern sehr hoch und variieren auch auf nationaler Ebene; dies ist auf eine sehr unterschiedliche Beteiligung der öffentlichen Hand an den Kita-Kosten zurückzuführen (Stern et al. 2015).

Mehr Betreuungsplätze zu schaffen wird als Notwendigkeit für die Vereinbarkeit von Familie und Beruf gesehen, meistens mit Blick auf die Erwerbstätigkeit der Mütter. Die Vereinbarkeit von Beruf und Familie ist ein zentrales Argument für den Bedarf an Kitas während der ganzen Zeitspanne von 100 Jahren des Bestehens des nationalen Verbandes der Kitas hinweg (Grob-Menges 2009). Zahlreiche Studien weisen in Modellrechnungen oder Choice-Experimenten nach, dass sich Beiträge der öffentlichen Hand an Kitas wirtschaftlich lohnen würden: Würden die Elternbeiträge gesenkt, würden mehr Mütter eine Erwerbstätigkeit aufnehmen 
oder ihre Erwerbstätigkeit erhöhen. Die kontinuierliche Teilhabe am Berufsleben würde ihre Laufbahnaussichten stärken und die Altersvorsorge verbessern, was sich in höheren Steuereinnahmen und geringeren Sozialkosten niederschlagen würde (Jeanrenaud et al. 2019). Besonders für Frauen, die in Berufen mit niedrigem Einkommen tätig sind, lohnt es sich in der Schweiz finanziell nicht, die Berufstätigkeit fortzuführen und die Kinder in der Kita betreuen zu lassen (Giudici und Schumacher 2017). Eine stärkere Beteiligung der Frauen in der Erwerbsarbeit geht mit einer egalitäreren Aufteilung der Aufgaben der Kinderbetreuung einher (BfS 2017, S. 40). Entsprechend wird die Forderung nach einer „Erweiterung öffentlich-privater Partnerschaften zur Finanzierung der Kinderbetreuungsangebote“ (Burger et al. 2017, S. 17) gestellt. Weiter wird argumentiert, dass sich die Investitionen in die frühkindliche Bildung und Betreuung nicht nur wegen der wirtschaftlichen Vorteile auszahlen, sondern auch wegen der Chancen für eine positive Entwicklung des Kindes durch die Angebote: „Dabei ist zu beachten, dass stets das Interesse des Kindes und nicht das der Wirtschaft im Zentrum stehen muss. Kinderbetreuungsangebote dürfen nicht primär im Dienst des Arbeitsmarkts stehen, sondern sollen Eltern in ihren Betreuungs- und Erziehungsaufgaben unterstützen und auf diese Weise eine positive Entwicklung des Kindes begünstigen und das Wohl des Kindes sicherstellen“ (Burger et al. 2017, S. 19). Während die Kita mit dem Vereinbarkeitsthema weiterhin einen wichtigen Aspekt der Gleichstellungspolitik darstellen, werden sie im aktuellen Diskurs nicht mehr wie vor hundert Jahren nur als „Nothilfe“, sondern als positiver Einflussfaktor für die Entwicklung des Kindes gesehen und damit auch als Bildungsinstitutionen verstanden. Aufgrund der engen Verwobenheit der Kita mit den Familienidealen, Vorstellungen von Mütterlichkeit, und der zugleich bestehenden Forderung nach einer grösseren Gleichstellung der Geschlechter erweist sich die Kita als eine stark in Veränderung begriffene, für Gleichstellung wie auch für Vorstellungen von Geschlechterdifferenzen relevante Organisation: es kann vermutet werden, dass doing und undoing gender auch in den Alltagspraktiken von Kitas zum Tragen kommt und die organisationalen Bedingungen einen wichtigen Einblick in die Konstruktion und Performanz von gender erlaubt.

\subsubsection{Der Beruf der Kinderbetreuung als historisch weiblich konnotiert}

Die Kindertagesstätten Ende des 19. Jahrhundert und in der ersten Hälfte des 20. Jahrhunderts wurden ausschliesslich von Frauen geführt. Frauen wurden aufgrund ihres Geschlechts als geeignet angesehen, kleine Kinder anstelle der Mutter 
in der familienergänzenden Kinderbetreuung zu betreuen. Frauen sind zugleich Mütter oder können Mutter werden, während Männer von diesen Tätigkeiten ausgeschlossen wurden. Obwohl es Pädagogen waren, die die Konzepte für die Kita erarbeiteten, beispielsweise Wolke mit der Idee von „Spielzimmern“ und „Denklernzimmern“, oder Fröbel mit dem Aufbau von Kindergärten und seinen Überlegungen zu „Spielgaben“, waren die in den Kitas tätigen Erwachsenen meistens Frauen. Mit Rückbezug auf Pestalozzi wurde postuliert, dass die Mutter dem Kind die beste frühe Bildung angedeihen würde (Reyer und Franke-Meyer 2016) und die Betreuung von kleinen Kindern darum von Frauen übernommen werden sollte. Die familienähnliche Gestaltung der Kita galt als das zentrale Qualitätsmerkmal, die Kita soll nicht wie eine Anstalt, sondern wie eine familiärer Wohnraum gestaltet sein (Kasüschke 2016). Die Betonung der Betreuenden in der Kita als Ersatz für die Mutter zu Hause zeigt sich auch in Bezeichnungen wie „Kindergartentante“ (Schütz 2019), die eine familiale Beziehung implizieren. Betreuung von kleinen Kindern ist demnach nicht nur aufgrund des zahlenmässig sehr hohen Anteils an Frauen im Beruf ein weiblich konnotierter Beruf, sondern auch weil Kinderbetreuung - und besonders diejenige von Kleinkindern - als naturgegebene Fähigkeit und Aufgabe von Frauen verstanden wurde.

Diese kulturhistorischen Wurzeln der (Klein-)Kinderbetreuung und ihre Verknüpfung mit Weiblichkeit prägt auch heute noch das Berufsfeld. Das zeigt auch der Vergleich mit dem Lehrberuf. Historisch gesehen ist der Lehrberuf ein Männerberuf, auch wenn, insbesondere in den ersten Schuljahren, aktuell ein sehr geringer Anteil von Männern den Beruf ausübt. Waren es zuerst ausschliesslich Männer, die den Lehrberuf ausübten, änderte sich dies Ende des 19. Jahrhunderts mit Einführung der allgemeinen Schulpflicht und den hierdurch eingeführten Bildungsangeboten für Mädchen. Die Mädchen und die jüngeren Kinder sollten von Frauen als Lehrpersonen unterrichtet werden, der Beruf öffnete sich in Konsequenz auch für Frauen. Ungleichheiten aufgrund des Geschlechts bestanden jedoch bis Mitte des 20. Jahrhunderts, so zum Beispiel Lohnunterschiede, das Zölibatsgebot für Lehrerinnen - Lehrerinnen wurden mit der Heirat vom Schuldienst ausgeschlossen - sowie die praktizierte Regulierung des Arbeitsmarktes durch Entlassungen (bei Lehrpersonenüberfluss) oder Einstellungen (bei Lehrpersonenmangel) von Frauen. Im Bildungssystem zeigt sich hier ein historisches doing gender: Bildung älterer Kinder galt als Männerberuf und ist mit höherem Status versehen, Bildung, Erziehung und Betreuung von Kleinkindern ist ein Frauenberuf mit niedrigerem Status.

Diese historischen Einflüsse können als eine Erklärung für die noch heute vorgefundene Trennung von „Bildung“ und „Betreuung“ herangezogen werden. Auch wenn Betreuung und Bildung besonders in der frühen Kindheit nicht 
getrennt werden können - und auch nicht getrennt werden sollen (Vogt 2015) - ist diese Unterscheidung sowohl strukturell als auch wirtschaftlich und kulturell verankert. In der Schweiz wird die Sicherung der Betreuung in der Kita dem Bereich des Sozialen zugewiesen, der Kindergarten gehört zur Schule und damit zum Bildungsbereich. Betreuung wird mit geringeren Anforderungen an beruflicher Qualifikation verbunden und tiefer entlohnt. Betreuung wird nach wie vor weiblich konnotiert und als eine Tätigkeit angesehen, für die eine Frau ,natürlicherweise", also lediglich aufgrund ihres Geschlechts kompetent ist und die nur ein geringes Mass an Ausbildung erfordert.

Auch in Bezug auf die professionellen Akteurinnen und Akteure erweist sich die Kita als ein besonders interessantes und dynamisches Feld, in welchem Prozesse und Aushandlungen von Geschlecht, doing und undoing gender, stattfinden. Als traditionell weibliche und wenig professionalisierte Institutionen, wird die Kinderbetreuung nach und nach professionalisiert und der Beruf auch für Männer zugänglich. $15.8 \%$ der Lehrstellen zur „Fachperson Betreuung Kind“ wurden im Jahr 2018 mit Männern besetzt (SavoirSocial 2020), was bedeutet, dass rund $85 \%$ der Kinderbetreuenden in Ausbildung Frauen sind. Rein aufgrund des Mengenverhältnisses handelt es sich bei der Kinderbetreuung demnach um einen Frauenberuf. Der aktuelle Männeranteil hat jedoch über die letzten zehn Jahren eine Verdoppelung erfahren: im Jahr 2008 waren nur 7,3 \% der Einsteigenden in die Lehre der „Fachperson Betreuung Kind“ Männer (SavoirSocial 2018). Die Zahl der Kitas steigt, damit auch der Bedarf an Beschäftigten in den Kitas. In vielen Ländern Europas zeigt sich eine Zunahme von Männern, die in Kitas arbeiten, allerdings nicht in allen. Bis anhin stellten Männer in keinem der untersuchten europäischen Ländern mehr als zehn Prozent der Beschäftigten (Rohrmann 2020). Hinsichtlich dieser Zunahme männlicher Kinderbetreuer erweist sich die Kita als ein hoch dynamisches Feld, in dem gender aktuell intensiv verhandelt wird.

Die Kitas erweisen sich auch im Hinblick auf die Kinderbetreuenden als gendered organisations (Acker 1990), vergeschlechtlichte Organisationen, in denen die Kultur, Prozesse und Strukturen nicht geschlechtsneutral, sondern an einem bestimmten Ideal einer weiblichen Mitarbeiterin ausgerichtet sind. Mit Blick auf Kindergärten sagt Rohrmann (2009, S. 34) provokativ: „Die Gärten der Kinder sind die Gärten der Frauen" und bezieht sich dabei auf die Dominanz der Frauen bei den Fachpersonen wie auch auf die Raumgestaltung. Männer sind in der Kinderbetreuung nicht nur in der Minderheit, ihre Anwesenheit wird auch negativ eingeschätzt. Kinderbetreuer werden wegen ihres Geschlechts unter Pädophilie-Verdacht gestellt (Rohrmann 2009), sie befinden sich häufig in einem Spannungsfeld „Zwischen Idealisierung und Verdächtigung“ (Rohrmann 2014, S. 67). 
Der hohe Frauenanteil wie auch die historische Entwicklung des Berufs machen die Kinderbetreuung zu einem Frauenberuf. In den letzten Jahren stieg der Anteil der Männer als Kinderbetreuer. Dennoch ist das Idealbild der Kinderbetreuenden, das Bild des ideal workers (Acker 1990) das Bild einer Frau, einer Kinderbetreuerin. Kinderbetreuer sind mit Verdächtigungen konfrontiert. Für die Erforschung von doing und undoing gender ist darum die Kita von grossem Interesse, auch in Bezug auf die Idealbilder des Berufs und den Praktiken von Gleichheit und Differenz von Frauen und Männern in diesem Beruf, der so stark weiblich konnotiert ist.

Auf der Grundlage der theoretischen Zugänge zu doing und undoing gender und den Besonderheiten des organisationalen Kontextes der Kitas wurden im Forschungsprojekt „Puppenstuben, Bauecken und Waldtage: (Un)doing gender in der Kita“ vielfältige methodische Zugänge gewählt und mit einer ethnografischen Forschungsstrategie miteinander verbunden. Im dritten Teil dieses Kapitels stellen wir das Forschungsdesign und die Methoden im Überblick dar.

\section{3 (Un)doing gender empirisch: Forschungsdesign und Methoden}

Aufbauend auf den im ersten Teil dieses Kapitels besprochenen ethnomethodologischen, diskursiven und praxistheoretischen Konzeptionen von doing und undoing gender haben wir im Projekt „Puppenstuben, Bauecken und Waldtage: (Un)doing gender in der Kita" Geschlecht als diskursive, soziale und materielle Praxis untersucht. Dafür eignet sich ein qualitatives Vorgehen, das die Beobachtung von Praktiken und die diskursive Ebene in Interaktionen, Gesprächen und Dokumenten verbindet (Bruni et al. 2005; Gherardi und Poggio 2007; Wrana 2012). Als Forschungsstrategie wurde ein ethnografisches Vorgehen gewählt, das es erlaubt, im Wechsel von Datensammlung und Analyse den verschiedenen Facetten der gewählten Forschungsfoki nachzugehen sowie verschiedene Erhebungsmethoden zielgerichtet zu kombinieren (Breidenstein et al. 2020; Flick 2001; Gherardi 2019; Hammersley 2006; Skeggs 2001; Zinnecker 2000).

Das Projekt setzte sich zum Ziel, Konstruktionen von Geschlecht in der frühkindlichen Bildung und Betreuung auf den analytischen Ebenen der institutionellen Arrangements (Goffman 1994), den symbolisch-kulturellen Ressourcen (Gherardi 1994, 1995; Wetterer 2003, 2006) sowie der Interaktionen (West und Zimmerman 1987) und Alltagspraktiken (Bruni et al. 2004, 2005) zu untersuchen. Bezugnehmend auf die im vorherigen Kapitel dargestellten Konstellationen in der 
historischen Entwicklung und dem Geschlechterverhältnis bei den Kinderbetreuenden, bieten sich die Kitas als ein äusserst facettenreiches Forschungsfeld für dieses Vorhaben an. Die folgenden drei Forschungsfoki standen im Zentrum:

1. Institutionelle Arrangements: Auf welche Weisen wird Geschlecht in den pädagogischen Konzepten, Ausbildungsinhalten, Verständnissen von professionellem Handeln und Wissen und im räumlichen Angebot der Kita konstruiert?

2. Interaktionen: Welche Formen von doing und undoing gender zeigen sich in den Alltagspraktiken der Kita, in den Interaktionen zwischen Erziehenden und Kindern, zwischen Kindern und dem jeweiligen Raum- und Spielangebot sowie den angebotenen Aktivitäten?

3. Männer als Kinderbetreuer: Inwieweit bringen Männer in der Kita etwas „Anderes" in die Alltagsarbeit ein? Welchen Einfluss hat dies auf die bestehende organisationale Praxis?

\subsubsection{Forschungsdesign}

Das Forschungsdesign umfasste zur Analyse der institutionellen Arrangements eine Dokumentenanalyse der Ausbildungsmanuale für angehende Kinderbetreuende, Interviews mit Kita-Leitungen und Kinderbetreuenden zu ihren professionellen Selbstverständnissen, sowie Raumanalysen mittels visueller Ethnografie (Fotos und Raumskizzen) (Pink 2008). Für den zweiten, auf Interaktionen und Alltagspraktiken zielenden Forschungsfokus stand Beobachtung im Zentrum. Dabei wurde der Alltag in den Kitas beobachtet und mittels Feldnotizen protokolliert wie auch videografiert. Für den dritten Fokus, Männer als Kinderbetreuer, wurden Interviews, Beobachtungen und Videografie eingesetzt. Die verschiedenen Forschungsfragen wurden somit jeweils mit mehreren qualitativen Verfahren untersucht. Dies ermöglichte eine Triangulation (Flick 2008): verschiedene Ebenen der Konstruktion von Geschlecht, die diskursive Ebene der Konstruktion von Geschlechterwissen und die performative Ebene des Alltagshandeln konnten erfasst und miteinander in Verbindung gesetzt werden. Durch das ethnografische Vorgehen werden die an den Interaktionen und Interpretationen beteiligten Personen als aktive Akteurinnen und Akteure, Interpretinnen und Interpreten wahrgenommen, die in der Auseinandersetzung mit gesellschaftlichen Normen, Deutungssystemen und Strukturen in der alltäglichen Arbeit mit Kindern doing und undoing gender praktizieren.

Ethnografische Forschungsprozesse umfassen verschiedene methodische Zugänge und eine zeitliche Aufteilung in Phasen der Datenerhebung im Feld, 


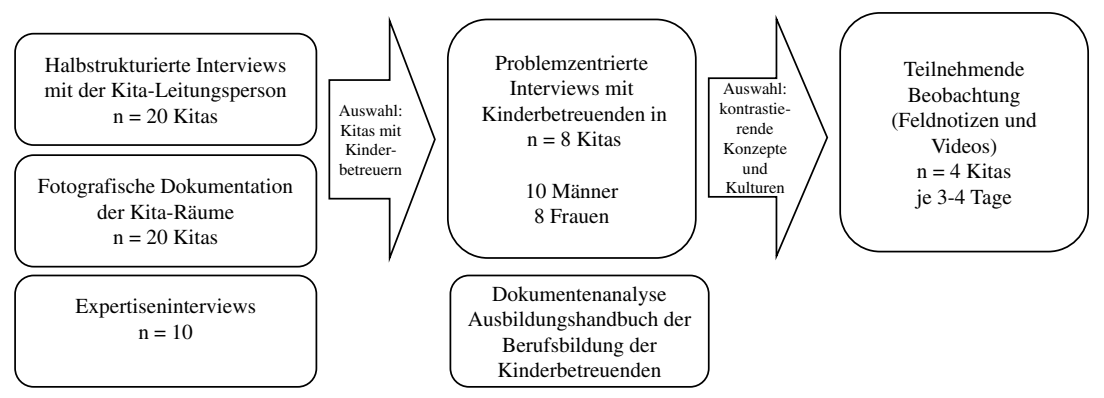

Abb.2.1 Forschungsdesign „Puppenstuben, Bauecken und Waldtage: (Un)doing Gender in der Kita“"

gefolgt von Analysen, die jeweils wieder in die nächste Phase der Datenerhebung im Feld einfliessen (Skeggs 2001). Der Forschungsprozess kann in insgesamt drei Phasen unterteilt werden (Abb. 2.1). In der ersten Phase haben wir den Feldkontakt aufgebaut und insgesamt 20 Kitas besucht, die Leitungsperson interviewt und uns die räumliche Situation zeigen lassen. In einer zweiten Phase standen die in diesen Kitas arbeitenden Kinderbetreuer und Kinderbetreuerinnen im Fokus, mit denen wir insgesamt 18 Interviews führten. In der dritten und letzten Phase beobachteten wir Interaktionen und Alltagspraktiken in vier Kitas, die aufgrund der durch die vorangegangenen Phasen nun vorhandenen detaillierteren Informationen nun gezielt ausgewählt werden konnten.

\subsubsection{Stichproben, Datenerhebung und -analyse}

Männer sind in den Kitas in der Minderheit. 2011, zu Beginn der Studie, wurden nur 9.2 \% der Lehrverträge für Fachpersonen Betreuung Kind mit Männern abgeschlossen (SavoirSocial 2020). Es war deshalb entscheidend, Kitas zur Teilnahme am Forschungsprojekt zu gewinnen, in denen auch Männer als Kinderbetreuende tätig waren. In Hinblick auf die Forschungsfragen nach dem professionellen Verständnis der Kinderbetreuenden wurden spezifisch Kitas gesucht, in denen Männer mit abgeschlossener Ausbildung als Fachperson Betreuung arbeiteten. „Fachperson Betreuung“ ist seit der Reform der dreijährigen Ausbildung mit eidgenössischem Fähigkeitszeugnis im Jahr 2005 die für die Deutschschweiz geltende Berufsbezeichnung für Kinderbetreuende (SBFI 2005), Dieser Beruf entspricht der in Deutschland gängigen Bezeichnungen Erzieher und Erzieherin. 
Wir rekrutierten Kitas über die Kontakte einer Webseite, die für mehr Männer in der Kinderbetreuung sensibilisierte ${ }^{1}$, über die Anfrage bei Netzwerken von Kitas sowie im direkten Kontakt mit Kitas. Zudem führten wir zehn Interviews mit Experten und Expertinnen für die Deutschschweizer Kita-Landschaft, die uns zu einem Überblick über relevante Dokumente, Akteurinnen und Akteure sowie wichtige historische Entwicklungen verhalfen.

Für die erste Phase des Forschungsprojektes konnten insgesamt 20 Kitas gewonnen werden. In diesen Kitas wurden mit der Kita-Leitung (15 Frauen und 5 Männer) halbstrukturierte Interviews geführt. Zudem zeigten uns die Leitungen die Räumlichkeiten der Kitas, die Erläuterungen während dieser Raumbegehungen wurden digital aufgenommen und es wurden Fotos der Räume und Raumskizzen gemacht. Für die Raumanalyse standen 858 Fotos zur Verfügung.

Für die zweite Phase wurden die acht Kitas, in denen ausgebildete männliche Kinderbetreuer tätig waren, für problemzentrierte Interviews (Witzel 1989) mit den männlichen Kinderbetreuern sowie jeweils einer weiblichen Kollegin angefragt. Dies, um in der gleichen Organisation den Eintritt des oftmals ersten Manns in die Kita aus Perspektive des Betroffenen, zugleich aber auch aus Perspektive einer anwesenden Kollegin erfassen zu können (Gherardi und Poggio 2007). Es wurden 18 Interviews mit zehn Kinderbetreuern und acht Kinderbetreuerinnen geführt.

Für die Dokumentenanalyse der Ausbildungsmaterialien wurden die Handbücher der Fachrichtung Kinderbetreuung gesichtet und das Kapitel aus den Materialien zur „Allgemeinen Berufskunde“ zum Thema Gender ausgewählt und analysiert.

Aus dem Teilsample der acht Kitas, die Männer als Kinderbetreuer beschäftigen, wurden vier für die Beobachtung und die Videobeobachtung in Phase drei ausgewählt. Die Selektion erfolgte hier zum einen aufgrund der Bereitschaft der Kitas, an der Studie weiter mitzuwirken, sowie der Kriterien des kontrastierenden Fallvergleichs: Kita Blau erschien uns interessant, da sie zum Erhebungszeitpunkt einen Männeranteil von rund $50 \%$ aufwies, was schweizweit eine Besonderheit darstellt. Kita Rot und Grün zeichnen sich durch eine klar formulierte pädagogische Schwerpunktsetzung aus, Kita Grün zudem die Besonderheit, dass sich die von einem Mann geleitete Gruppe ganztags im Freien aufhält. Auch Kita Gelb beschäftigte einen ausgebildeten Mann sowie weitere Praktikanten und erschien uns darüber hinaus sehr typisch für viele der untersuchten Kitas. In allen Kitas

\footnotetext{
${ }^{1}$ Die verwendete webseite: www.kinderbetreuer.ch ist nicht mehr online. Aktuell finden sich ähnliche Webseiten wie beispielsweise: https://www.kibesuisse.ch/kinderbetreuer/; https:// www.maenner.ch/mencare/mehr-maenner-in-die-kinderbetreuung-maki-3/; oder https://koo rdination-maennerinkitas.de/.
} 
wurde das schriftliche Einverständnis zur Videoaufzeichnung von Eltern sowie den gefilmten Betreuenden eingeholt.

Geplant waren in jeder Kita vier Beobachtungstage, in einer Kita konnte nur an drei Tagen beobachtet werden. An diesen 15 Beobachtungstagen in den Kitas wurden die Alltagsinteraktionen durch jeweils zwei Personen des Forschungsteams beobachtet und protokolliert sowie mittels zweier Videokameras videografiert. Es wurden insgesamt $50 \mathrm{~h}$ Videomaterial erhoben. Während eine Videokamera ad hoc eingesetzt werden konnte, folgte eine Forscherin jeweils im Sinne eines Shadowings (Czarniawska 2007) konsequent für jeweils einen halben Tag der männlichen, dann für die andere Hälfte des Tages der weiblichen Fachperson. Durch diese Aufteilung konnte sichergestellt werden, dass wir die Personen nicht zu lange mit der Beobachtung belasten, zugleich konnten wir so aber auch die gleiche Person in der verbleibenden Zeit aus einer grösseren Distanz beobachten. Mit der klaren Entscheidung, welche Person für eine bestimmte Zeitdauer bei all ihren Alltagsaktivitäten in der Kita beobachtet wird, werden systematisch variierende „Blickschneisen“ (Mohn 2018) möglich. Durch die bei diesem Vorgehen entstehende unmittelbare Nähe zur beobachteten Person, aber auch ihren Alltagstätigkeiten entwickelten sich ad hoc Gespräche, mit der die dazugehörigen Deutungs- und Sinnkonstruktionen zu für uns bedeutsamen Situationen näher erfragt werden konnten.

Eine zweite Forscherin hatte die Möglichkeit, mit einer zweiten Kamera andere Szenen zu filmen, oder aber offen zu beobachten. In dieser Rolle entschieden wir häufig ad hoc und je nach Aktivitäten in der Gruppe. Die hier gewählten „Blickschneisen“ (Mohn 2018) waren häufiger auf die Aktivitäten in der Gruppe, das Spiel der Kinder, oder auch Aktivitäten an bestimmten Orten wie z. B. der Garderobe oder dem Sandkasten ausgerichtet und folgten weniger der Perspektive der Kinderbetreuenden.

Für die Datenanalyse wurden entsprechend der ethnographischen Forschungsstrategie verschiedene Methoden eingesetzt. Das spezifische forschungsmethodische Vorgehen wird in jedem der in diesem Band versammelten Kapitel detailliert erläutert und begründet. An dieser Stelle wird mit Tab. 2.1 ein Überblick über die verschiedenen Zugänge zur Datenanalyse gegeben:

\subsection{Mehrperspektivische theoretische und analytische Zugänge}

Doing gender und undoing gender für eine empirische Untersuchung zu konzeptualisieren stellt, wie wir gesehen haben, einige Herausforderungen an die 
Tab. 2.1 Überblick über die forschungsmethodischen Zugänge der Buchkapitel

\begin{tabular}{l|l|l}
\hline Datenmaterial & Analysemethode & Kapitel \\
\hline Fotografische Raumanalyse & Visuelle Ethnografie und Inhaltsanalyse & 3 \\
\hline $\begin{array}{l}\text { Videodaten aus 4 Kitas an 15 } \\
\text { Beobachtungstagen, } \\
50 \text { h Videomaterial }\end{array}$ & $\begin{array}{l}\text { Videobasierte Ethnografie, } \\
\text { Mikroanalyse der Interaktionen } \\
\text { Kinderbetreuende/r - Kind }\end{array}$ & 4 \\
\hline $\begin{array}{l}\text { Teilnehmende Beobachtungen } \\
\text { (Protokolle und Videomaterial) }\end{array}$ & $\begin{array}{l}\text { Praxistheoretische Analyse der } \\
\text { Rhythmen und Routinen }\end{array}$ & 5 \\
\hline $\begin{array}{l}\text { Problemzentrierte Interviews mit } \\
\begin{array}{l}\text { 10 Kinderbetreuern und } \\
8 \text { Kinderbetreuerinnen }\end{array}\end{array}$ & $\begin{array}{l}\text { Diskursanalyse der } \\
\text { Subjektpositionierungen männlicher } \\
\text { Kinderbetreuer }\end{array}$ & 6 \\
\hline $\begin{array}{l}\text { Videodaten aus 4 Kitas an 15 } \\
\text { Beobachtungstagen, } \\
50 \text { h Videomaterial }\end{array}$ & $\begin{array}{l}\text { Videobasierte, multimodale Analyse } \\
\text { der Männlichkeitskonstruktionen in } \\
\text { körperlichen Praktiken }\end{array}$ & 7 \\
\hline $\begin{array}{l}\text { Ausbildungshandbücher } \\
\text { Kinderbetreuung }\end{array}$ & $\begin{array}{l}\text { Dokumentenanalyse basierend auf der } \\
\text { Sozialwissenschaftlichen Hermeneutik }\end{array}$ & 8 \\
\hline
\end{tabular}

Forschenden. (Un)doing gender muss als performativ und damit fluide, zugleich aber als omnirelevant in den Blick genommen werden. Gender steht als Ressource immer zur Verfügung, wird jedoch nicht immer genutzt. Genderismen sind in diskursiven, sozialen und materiellen Praktiken konstruiert, herausgefordert und umgedeutet. Für die empirische Analyse von (un)doing gender stehen zwar konzeptionelle Überlegungen verschiedener Forschungstraditionen zur Verfügung, die jedoch keine standardisierten Methoden oder Analyseraster vorgeben. Hier verschiedene Möglichkeiten aufzuzeigen war Ziel des Forschungsprojekts „(Un)doing Gender in der Kita“, die gewählte ethnografische Forschungsstrategie und das damit verbundene multimethodische Design ermöglichte es uns, verschiedene analytische Zugänge zu (un)doing gender zu entwickeln und zu erproben. Kindertagesstätten erwiesen sich dabei als äusserst relevantes Feld. Sowohl in der historischen Entwicklung wie auch in den aktuellen Diskursen und Idealbildern wird Geschlecht auf unterschiedliche Weisen verhandelt. Zudem ist durch die Aktualität und Relevanz der Diskussion um pädagogische Qualität in der frühen Kindheit eine hohe Praxisrelevanz gegeben.

In der Pädagogik der frühen Kindheit wird der Gestaltung des Raumes eine grosse Bedeutung zugemessen. Franziska Vogt stellt im dritten Kapitel die Erforschung der räumlichen Arrangements mit visueller Ethnografie dar. Das für dieses Forschungsprojekt entwickelte Vorgehen erkundet die institutionalisierten Geschlechterarrangements im Raum und in den Artefakten wie Spielmaterial. 
Videoaufnahmen der alltäglichen Interaktionen zwischen Fachpersonen und Kindern werden von Franziska Vogt auf der Mikroebene hinsichtlich doing und undoing gender in Kap. 4 untersucht. Als Analyseinstrument dient eine aus den verschiedenen Theorien entwickelte Vorgehensweise, die doing und undoing gender als Differenz oder Gleichheit betonend versteht, und den interaktionalen Moment von Dramatisierung oder Dethematisierung in die Analyse einbezieht. Die gendered practices stehen im Zentrum des fünften Kapitels. Julia Nentwich arbeitet auf der Grundlage der ethnografischen Beobachtungsprotokollen sowie des Videomaterials heraus, wie die habituellen Routinen vergeschlechtlicht sind. Die Kontrastierung zwischen den beobachteten Routinen und Rhythmen in den vier Kitas erlaubt, die unterschiedlichen Taktgeber zu identifizieren.

Männer, die als Kinderbetreuer in einer Kita tätig sind, sehen sich mit verschiedenen Ansprüchen konfrontiert: sie sollen zugleich wie alle Kinderbetreuenden sein und typisch Männliches einbringen. Die Analysen der Interviews mit den Kinderbetreuern von Julia Nentwich und Wiebke Tennhoff in Kap. 6 zeigen, wie fluide und wechselnd verschiedene Subjektpositionen eingenommen werden. Das diskursanalytische Vorgehen erlaubt es, die verschiedenen Spielarten von doing und undoing gender herauszuarbeiten. Auf der Basis des Konzeptes des Habitus und mithilfe der theoretischen Zugänge zu doing masculinity analysiert Tobias Bockstaller in Kap. 7 die körperlichen Praktiken der Kinderbetreuer. Er basiert seine Analyse auf den Videos und untersucht, inwiefern die Kinderbetreuer in ihren körperlichen Praktiken verschiedene Männlichkeiten herstellen oder unterlaufen.

Die Ausbildungsmaterialien für die Fachpersonen Betreuung bilden das Datenmaterial der Analyse von Julia Wustmann in Kap. 8. Mittels sozialwissenschaftlicher Hermeneutik wird das Geschlechterwissen in den Ausbildungsunterlagen rekonstruiert. Bezüglich doing und undoing gender wird ein Spannungsfeld zwischen einem essentialistischen und einem konstruktivistischen Verständnis von Geschlecht und gender identifiziert. Im abschliessenden Kapitel fassen Julia Nentwich und Franziska Vogt den Beitrag des Forschungsprojektes zur Theoriebildung von doing und undoing gender sowie den multimethodischen und multiperspektivischen empirischen methodologischen Zugängen zusammen. Anhand der aus dem Forschungsprojekt hervorgehenden Praxisinterventionen skizzieren sie das Potenzial für die Praxis: Was bedeuten die Befunde für die Alltagspraxis in den Kitas? Wie können Männer als Kinderbetreuer stärker inkludiert und auf welche Weise können Organisationsentwicklungsprozesse das (un)doing gender in der Kita verändern? 


\section{Literatur}

Aumais, Nancy (2017). Studying the Doing and Undoing of Gender in the Organisation: Promises and Challenges. International Journal of Work Innovation, 2(2/3), 216-230.

Acker, Joan (1990). Hierarchies, Jobs and Bodies: Towards a Theory of Gendered Organizations. Gender and Society, 4(2), 139-158.

Beck, Ulrich, Bonss, Wolfgang, \& Lau, Christoph (2001). Theorien reflexiver Modernisierung- Fragestellungen, Hypothesen, Forschungsstrategien. In Ulrich Beck \& Wolfgang Bonss (Hrsg.), Die Modernisierung der Moderne (S. 11-59). Frankfurt a. M.: Suhrkamp.

Berger, Peter L., \& Luckmann, Thomas (1996). Die gesellschaftliche Konstruktion der Wirklichkeit: Eine Theorie der Wissenssoziologie [The Social Construction of Reality]. (Monika Plessner, Übers.). Frankfurt am Main: Fischer. (englisches Original 1966 erschienen).

Billig, Michael (1999a). Conversation Analysis and the Claims of Naivety. Discourse \& Society, 10(4), 572-576.

Billig, Michael (1999b). Whose Terms? Whose Ordinariness? Rhetoric and Ideology in Conversation Analysis. Discourse \& Society, 10(4), 543-558.

Breidenstein, Georg, Hirschauer, Stefan, Kalthoff, Herbert, \& Nieswand, Boris (2020). Ethnografie: Die Praxis der Feldforschung. Stuttgart: UTB.

Bruni, Attilla, Gherardi, Silvia, \& Poggio, Barbara (2004). Doing Gender, Doing Entrepreneurship: An Ethnographic Account of Intertwined Practices. Gender Work and Organization, 11(4), 406-429.

Bruni, Attilla, Gherardi, Silvia, \& Poggio, Barbara (2005). Gender and Entrepreneurship. An Ethnographic Approach. London/New York: Routledge.

Burger, Kaspar, Neumann, Sascha, \& Brandenberg, Kathrin (2017). Studien zur frühkindlichen Bildung, Betreuung und Erziehung in der Schweiz. Zusammenfassung der Bestandsaufnahme und politische Empfehlungen. Jacobs Foundation. https://jacobsfoundation.org/ app/uploads/2017/07/JF_Whitepaper_FBBE_kurz_deu-1.pdf. Zugegriffen: 30. Juni 20.

Bundesamt für Statistik (BFS) (2017). Familien in der Schweiz. Statistischer Bericht 2017. Neuchâtel: Bundesamt für Statistik. https://www.bfs.admin.ch/bfs/de/home/statistiken/ kataloge-datenbanken/publikationen.assetdetail.2347880.html. Zugegriffen: 30. Juni 20.

Bundesamt für Statistik (BFS) (2019). Nutzung von familienergänzender Kinderbetreuung, 2018. Neuchâtel: Bundesamt für Statistik. https://www.bfs.admin.ch/bfs/de/home/sta tistiken/bevoelkerung/familien/familienergaenzende-kinderbetreuung.assetdetail.105 07370.html. Zugegriffen: 30. Juni 20.

Butler, Judith (1988). Performative Acts and Gender Constitution: An Essay in Phenomenology and Feminist Theory. Theatre Journal, 40(4), 519-531.

Butler, Judith (1989). Foucault and the Paradox of Bodily Inscriptions. The Journal of Philosophy, 86(11), 601-607.

Butler, Judith (1991). Das Unbehagen der Geschlechter [Gender Trouble: Feminism and the subversion of identity]. (17. Aufl., Katharina Menke, Übers.). Frankfurt am Main: Suhrkamp. (englisches Original erschienen 1990). 
Butler, Judith (2009). Die Macht der Geschlechternormen und die Grenzen des Menschlichen [Undoing Gender]. (Karin Wördemann, \& Martin Stempfhuber, Übers.) Frankfurt am Main: Suhrkamp. (englisches Original erschienen 2004).

Chowdhury, Nilima, \& Gibson, Kerry (2019). This is (still) a Man's World: Young Professional Women's Identity Struggles in Gendered Workplaces. Feminism \& Psychology, 29(4), 475-493.

Connell, Raewyn (1987). Gender and Power. Society, the Person and Sexual Politics. Standford: Stanford University Press.

Czarniawska, Barbara, \& Sevón, Guje (2005). Global Ideas: How Ideas, Objects and Practices Travel in the Global Economy. Malmo: Copenhagen Business School.

Czarniawska, Barbara (2007). Shadowing and Other Techniques for Doing Fieldwork in Modern Societies. Copenhagen: Copenhagen Business School Press.

Davies, Bronwyn (2006). Subjectification: The Relevance of Butler's Analysis for Education. British Journal of Sociology of Education, 27(4), 425-438

Deutsch, Francine M. (2007). Undoing Gender. Gender \& Society, 21(1), 106-127.

Di Masso, Andrés, \& Dixon, John (2015). More Than Words: Place, Discourse and the Struggle over Public Space in Barcelona. Qualitative Research in Psychology, 12(1), 45-60.

Durrheim, Kevin (2012). Discourse, Action, Rhetoric: From a Perception to an Action Paradigm in Social Psychology. British Journal of Social Psychology, 51(3), 456-462.

Edley, Nigel, \& Wetherell, Margaret (1997). Jockeying for Position: The Construction of Masculine Identities. Discourse \& Society, 8(2), 203-217.

Faulstich-Wieland, Hannelore (2001). Gender Mainstreaming im Bereich der Kindertagesstätten. In Gabriele Ginsheim \& Dorit Meyer (Hrsg.), Gender Mainstreaming. Neue Perspektiven für die Jugendhilfe (S. 121-132). Berlin: Stiftung SPI.

Faulstich-Wieland, Hannelore, Weber, Martina, \& Willems, Katharina (2004). Doing gender im heutigen Schulalltag. Empirische Studien zur sozialen Konstruktion von Geschlecht in schulischen Interaktionen. Weinheim: Juventa.

Fenstermaker, Sarah, \& West, Candace (2001). Doing Difference' revisited. Probleme, Aussichten und der Dialog in der Geschlechterforschung. Kölner Zeitschrift für Soziologie und Sozialpsychologie, 41, 236-249.

Fenstermaker, Sarah, \& West, Candace (2002). Doing Gender, Doing Difference: Inequality, Power, and Institutional Change. New York: Routledge.

Flick, Uwe (2001). Qualitative Sozialforschung - Stand der Dinge. Soziologie (2), 53-66.

Flick, Uwe (2008). Triangulation. Eine Einführung (2. Aufl.). Wiesbaden: VS Verlag für Sozialwissenschaften.

Fournier, Valérie, \& Smith, Warren (2006). Scripting Masculinity. Ephemera: Theory \& Politics in Organization, 6(2), 141-162.

Friis, Pia (2008). Männer im Kindergarten. Wie man sie anwirbt - und dafür sorgt, dass sie auch bleiben. Oslo: Kunnskapsdepartmentet. https://www.uibk.ac.at/psyko/forschung/ele mentar/literatur/friis_maenner_im_kindergarten.pdf. Zugegriffen: 30. Juni 20.

Garfinkel, Harold (1967). Studies in Ethnomethodology. Cambridge, UK: Polity Press.

Gherardi, Silvia (1994). The Gender We Think, the Gender we Do in our Everyday Organizational Lives. Human Relations, 47(6), 591-610.

Gherardi, Silvia (1995). Gender, Symbolism and Organizational Cultures. London: Sage. 
Gherardi, Silvia (2016). To Start Practice Theorizing Anew: The Contribution of the Concepts of Agencement and Formativeness. Organization, 23(5), 680-698.

Gherardi, Silvia (2019). How to Conduct a Practice-based Study: Problems and Methods. Cheltenham, UK: Edward Elgar.

Gherardi, Silvia, \& Poggio, Barbara (2007). Gendertelling in Organizations. Narratives from Male-Dominated Environments. Fredriksberg: Liber, Copenhagen Business School Press.

Gildemeister, Regine, \& Hericks, Katja (2012). Geschlechtersoziologie: Theoretische Zugänge zu einer vertrackten Kategorie des Sozialen. München: Oldenbourg Wissenschaftsverlag.

Gildemeister, Regine, \& Robert, Günther (2008). Geschlechterdifferenzierungen in lebenszeitlicher Perspektive: Interaktion - Institution - Biografie. Wiesbaden: VS Verlag für Sozialwissenschaften.

Gildemeister, Regine, \& Wetterer, Angelika (1992). Wie Geschlechter gemacht werden. Die soziale Konstruktion von Zweigeschlechtlichkeit und ihre Reifizierung in der Frauenforschung. In Gudrun-Axeli Knapp \& Angelika Wetterer (Hrsg.), TraditionenBrüche. Entwicklungen feministischer Theorie (S. 201-254). Freiburg im Breisgau: Kore

Giudici, Francesco, \& Schumacher, Reto (2017). Erwerbstätigkeit von Müttern in der Schweiz: Entwicklung und individuelle Faktoren. Social Change in Switzerland, (10), $1-13$.

Goffman, Erving (1976). Wir alle spielen Theater: die Selbstdarstellung im Alltag [The Presentation of Self in Everyday Life]. München: Piper. (englisches Original 1959 erschienen).

Goffman, Erving (1994). Das Arrangement der Geschlechter [The Arrangement Between the Sexes]. In Hubert A. Knoblauch (Hrsg. und Übersetz.), Interaktion und Geschlecht. New York: Campus Verlag. (englisches Original 1977 erschienen).

Grob-Menges, Ursula (2009). 100 Jahre Kinderbetreuung - und stets am Anfang. In SAGW (Hrsg.), Familienergänzende Betreuung, Erziehung und Bildung von Kindern - Ein Generationenprojekt in privater und staatlicher Verantwortung (S. 75.82). Bern: SAGW.

Gildemeister, Regine. (2017). Doing Gender: eine mikrotheoretische Annäherung an die Kategorie Geschlecht. In Beate Kortendiek, Birgit Riegraf,\& Katja Sabisch (Hrsg.), Handbuch Interdisziplinäre Geschlechterforschung (S. 409-417). Wiesbaden: VS Verlag für Sozialwissenschaften.

Hammersley, Martyn (2006). Ethnography: Problems and Prospects. Ethnography and Education, 1(1), 3-14.

Heintz, B. (2001). Geschlecht als Unordnungsprinzip. Entwicklungen und Perspektiven der Geschlechtersoziologie. Kölner Zeitschrift für Soziologie und Sozialpsychologie, 53, 9-29.

Heintz, Bettina, \& Nadai, Eva (1998). Geschlecht und Kontext, DeInstitutionalisierungsprozesse und geschlechtliche Differenzierung. Zeitschrift für Soziologie, 27(2), 75-93.

Hirschauer, Stefan (1994). Die soziale Fortpflanzung der Zweigeschlechtlichkeit. Kölner Zeitschrift für Soziologie und Sozialpsychologie, 46(4), 668-692.

Hirschauer, Stefan (2001). Das Vergessen des Geschlechts. Zur Praxeologie einer Kategorie sozialer Ordnung. Kölner Zeitschrift für Soziologie und Sozialpsychologie, Sonderheft, 41, 208-235. 
Hirschauer, Stefan (2012). Die Praxis der Geschlechter(in)differenz und ihre Infrastruktur. In Julia Graf, Kristin Ideler, \& Sabine Klinger (Hrsg.), Geschlecht zwischen Struktur und Subjekt: Theorie, Praxis, Perspektiven (S. 153-171). Opladen: Barbara Budrich.

Hirschauer, Stefan (2014). Un/doing Difference. Die Kontingenz sozialer Zugehörigkeiten. Zeitschrift für Soziologie, 43(3), 170-191.

Hirschauer, Stefan (2016). Judith, Niklas und das Dritte der Geschlechterdifferenz: undoing gender und die Post Gender Studies. GENDER - Zeitschrift Für Geschlecht, Kultur und Gesellschaft, 3, 114-129.

Höltershinken, Dieter \& Kasüschke, Dagmar (1996). Betriebliche Kinderbetreuung von 1875 bis heute. $v$ in Deutschland. Opladen: Leske + Budrich.

Hollander, Jocelyn A. (2013). "I Demand More of People": Accountability, Interaction, and Gender Change. Gender \& Society, 27(1), 5-29.

Horanyi, Rita (2014). Performance and Performativity. In Anthony Elliott (Ed.), Routledge Handbook of Social and Cultural Theory (pp. 74-96). Abingdon: Routledge.

Janssens, Maddy, \& Steyaert, Chris (2019). A Practice-Based Theory of Diversity: Respecifying (In)Equality in Organizations. Academy of Management Review, 44(3), 518-537.

Jeanrenaud, Claude, Kis, Alexandra, Gnaegi, Philippe, \& Soulet, Marc-Henry (2019). Etudier un nouveau mode de financement des structures d'accueil basé sur les incitations et favoriser ainsi la socialisation des jeunes enfants. Bern: ProFamilia. https://www. profamilia.ch/tl_files/Dokumente/Dokumente_News/MM_23.05.2019_Gesamtbericht\% 20101\%20Seiten\%20(nur\%20auf\%20Franzoesisch).pdf. Zugegriffen: 30. Juni 20.

Joris, Elisabeth, \& Witzig, Heidi (1995). Brave Frauen, aufmüpfige Weiber: Wie sich die Industrialisierung auf Alltag und Lebenszusammenhänge von Frauen auswirkte (18201940). Zürich: Chronos.

Joris, Elisabeth, \& Witzig, Heidi (2001). Frauengeschichte(n). Dokumente aus zwei Jahrhunderten zur Situation der Frauen in der Schweiz. Zürich: Limmatverlag.

Kasüschke, Dagmar (2016). Die Kindertageseinrichtung als Raum pädagogisch inszenierter Kindheit. In Rita Braches-Chyrek, \& Charlotte Röhner (Hrsg.), Kindheit und Raum (S.180-198). Opladen: Barbara Budrich.

Kelan, Elisabeth (2008). Gender, Risk and Employment Insecurity: The Masculine Breadwinner Subtext. Human Relations, 61(9), 1171-1202.

Kelan, Elisabeth K. (2009). Performing Gender at Work. Basingstoke: Palgrave Macmillan.

Kelan, Elisabeth K. (2010). Gender Logic and (Un)doing Gender at Work. Gender, Work \& Organization, 17(2), 174-194.

Kelle, Helga (2016). Herausforderungen ethnographischer Forschung zu Pädagogik und Geschlecht. In Ulrike Graff, Katja Kolodzig, \& Nikolas Johann (Hrsg.), Ethnographie - Pädagogik - Geschlecht: Projekte und Perspektiven aus der Kindheits- und Jugendforschung (S. 3-16). Wiesbaden: Springer Fachmedien Wiesbaden.

König, Tomke (2012). Familie heißt Arbeit teilen: Transformationen der symbolischen Geschlechterordnung. Konstanz: UVK Verlagsgesellschaft.

Koschorke, Albrecht., Ghanbari, Nacim, Esslinger, Eva, Susteck, Sebastian, \& Taylor, Michael T. (2010). Vor der Familie: Grenzbedingungen einer modernen Institution. Konstanz: University Press.

Lorber, Judith (2000). Using Gender to Undo Gender: A Feminist Degendering Movement. Feminist Theory, 1(1), 79-95. 
Lynch, Michael (2001). Ethnomethodology and the Logic of Practice. In Theodore R. Schatzki, Karin Knorr-Cetina, \& Eike von Savigny (eds.), The Practice Turn in Contemporary Theory (pp. 131-148). London: Routledge.

MacNaughton, Glenda (2000). Rethinking Gender in Early Childhood Education. London: Paul Chapman.

Maihofer, Andrea (2004). Was wandelt sich im aktuellen Wandel der Familie? In Joachim Beehorst, Alex Demirovic, \& Michael Guggemos (Hrsg.), Kritische Theorie im gesellschaftlichen Strukturwandel (S. 384-408). Frankfurt a.M.: Suhrkamp.

Martin, Patricia Y. (2001). "Mobilizing Masculinities": Women's Experiences of Men at Work. Organization, 8(4), 587-618.

Martin, Patricia Y. (2003). "Said and Done" versus "Saying and Doing”. Gendering Practices, Practicing Gender at Work. Gender \& Society, 17(3), 342-366.

Martin, Patricia Y. (2004). Gender As Social Institution. Social Forces, 82(4), 1249-1273.

Martin, Patricia Y. (2006). Practising Gender at Work: Further Thoughts on Reflexivity. Gender, Work \& Organization, 13(3), 254-276.

Meuser, Michael (2009). Männlichkeiten in Bewegung - Zur Aktualität des Konzepts der hegemonialen Männlichkeit angesichts des Wandels von Erwerbsarbeit. In Brigitte Aulenbacher \& Birgit Riegraf(Hrsg.), Erkenntnis und Methode, Geschlechterforschung in Zeiten des Umbruchs (S. 249-265). Verlag für Sozialwissenschaften.

Miller, Leslie J. (2000). The Poverty of Truth-Seeking: Postmodernism, Discourse Analysis and Critical Feminism. Theory \& Psychology, 10(3), 313-352.

Mohn, Bina E. (2018). Kamera-Ethnographie. In A. Geimer, C. Heinze, \& R. Winter (Hrsg.), Handbuch Filmsoziologie (S. 1-21). Wiesbaden: Springer.

Morison, Tracy, \& Macleod, Catriona (2013). A Performative-Performance Analytical Approach: Infusing Butlerian Theory Into the Narrative-Discursive Method. Qualitative Inquiry, 19(8), 566-577.

Müller, Marianne, Trede, Ines, Neumann, Jörg, \& Kriesi, Irene (2017). Arbeitnehmende im Sozialbereich: Ergebnisbericht. Zollikofen: SavoirSocial. https://savoirsocial.ch/wp-con tent/uploads/2019/12/SAVOIRSOCIAL_Bericht_Arbeitnehmendenbefragung_180130_ D.pdf. Zugegriffen: 30. Juni 20.

Müller, Ursula, Müller-Franke, Waltraud, Pfeil, Patricia, \& Wilz, Sylvia (2007). Zwischen De-Thematisierung und Vergewisserung: Geschlechterkonstruktionen im Organisationswandel am Beispiel Polizei. In Regine Gildemeister \& Angelika Wetterer (Hrsg.), Erosion oder Reproduktion geschlechtlicher Differenzierungen? Widersprüchliche Entwicklungen in professionalisierten Berufsfeldern und Organisationen (S. 32-56). Münster: Westfälisches Dampfboot.

Nentwich, Julia C. (2008). New Fathers and Mothers as Gender Troublemakers? Exploring Discursive Constructions of Heterosexual Parenthood and their Subversive Potential. Feminism \& Psychology, 18(2), 207-230.

Nentwich, Julia C., \& Morison, Tracy (2018). Performing the Self: Performativity and Discursive Psychology. In APA Handbook of the Psychology of Women: History, Theory, and Battlegrounds (Vol. 1, pp. 209-228). Washington: American Psychological Association.

Nentwich, Julia C., \& Kelan, Elisabeth K. (2014). Towards a Topology of 'Doing Gender': An Analysis of Empirical Research and Its Challenges. Gender, Work \& Organization, 21(2), 121-134. 
Nicolini, Davide (2013). Practice Theory, Work, and Organization an Introduction. Oxford: Oxford University Press.

Nicolini, Davide, \& Monteiro, Pedro (2017). The Practice Approach: For a Praxeology of Organisational and Management Studies. In Ann Langley \& Haridimos K. Tsukas (Eds.), The SAGE Handbook of Process Organization Studies (pp. 110-126). London: SAGE Publications

Ott, Marion, Langer, Antje, \& Rabenstein, K. (2012). Ethnographie und Diskursanalyse. In B. Friebertshäuser (Hrsg.), Feld und Theorie: Herausforderungen erziehungswissenschaftlicher Ethnographie (S. 169-184). Opladen: Budrich.

Pasero, Ursula (1995). Dethematisierung von Geschlecht. In Ursula Pasero \& Friederike Braun (Hrsg.), Konstruktion von Geschlecht (S. 50-66). Pfaffenweiler: Centaurus.

Pink, Sarah (2008). Doing Visual Ethnography. London: Sage.

Poggio, Barbara (2006). Editorial: Outline of a Theory of Gender Practices. Gender, Work \& Organization, 13(3), 225-233.

Potter, Jonathan, \& Wetherell, Margaret (1987). Discourse and Social Psychology: Beyond Attitudes and Behaviour. London: Sage.

Reckwitz, Andreas (2002). Toward a Theory of Social Practices: A Development in Culturalist Theorizing. European Journal of Social Theory, 5(2), 243.

Reyer, Jürgen (2006). Einführung in die Geschichte des Kindergartens und der Grundschule. Bad Heilbrunn: Julius Klinkhardt.

Reyer, Jürgen, \& Franke-Meyer, Diana (2016). Räume und Räumlichkeiten in der Geschichte der Pädagogik der frühen Kindheit und des Kindergartens. In Rita Braches-Chyrek, \& Charlotte Röhner (Hrsg.), Kindheit und Raum (S.163-179). Opladen: Barbara Budrich.

Ridgeway, Cecilia L., \& Correll, Shelly J. (2004). Unpacking the Gender System: A Theoretical Perspective on Gender Beliefs and Social Relations. Gender \& Society, 18(4), 510-531.

Risman, Barbara J. (2009). From Doing to Undoing: Gender as We Know It. Gender \& Society, 23(1), 81-84.

Rohrmann, Tim (2009). Gender in Kindertageseinrichtungen, ein Überblick über den Forschungsstand. München: DJI.

Rohrmann, Tim (2014). Männer in Kitas: Zwischen Idealisierung und Verdächtigung. In Jürgen Budde, Christine Thon, \& Katharina Walgenbach (Hrsg.), Männlichkeiten. Geschlechterkonstruktionen in pädagogischen Institutionen (S. 67-84). Opladen: Barbara Budrich.

Rohrmann, Tim (2020). Men as Promoters of Change in ECEC? An International Overview. Early Years, 40(1), 5-19.

Roßbach, Hans-Günther, \& Grell, Frithjof. (2012). Vorschulische Einrichtungen. In Uwe Sandfuchs, Wolfgang Melzer, Bernd Dühlmeier, \& Adly Rausch (Hrsg.), Handbuch Erziehung (S. 332-338). Bad Heilbrunn: Klinkhardt.

Salih, Sara (2002). Judith Butler. London: Taylor \& Francis.

SavoirSocial (2018). Berufsbildung im Sozialbereich: Männerquoten. https://savoirsocial.ch/ wp-content/uploads/2020/02/M\%C3\%A4nneranteile_SozialeBerufe_200211.pdf. Zugegriffen: 30. Juni 20.

SavoirSocial. (2020). Männeranteile Soziale Berufe. https://savoirsocial.ch/wp-content/upl oads/2020/02/M\%C3\%A4nneranteile_SozialeBerufe_200211.pdf. Zugegriffen: 30. Juni 20. 
Schegloff, Emanuel A. (1997). Whose Text? Whose Context? Discourse \& Society, 8(2), $165-187$.

Schütz, Julia. (2019). Die Tanten-Metapher. Neue Praxis, 2019(4), 293-303.

Shapiro, Michael J. (1992). Reading the Postmodern Polity: Political Theory as Textual Practice. Minneapolis: University of Minnesota Press.

Skeggs, Beverley (2001). Feminist Ethnography. In Paul Atkinson, Amanda Coffey, Sara Delamont, John Lofland, \& Lyn Lofland (Eds.), Handbook of Ethnography (pp. 426-442). London: Sage.

Staatssekretariat für Bildung, Forschung und Innovation (SBFI) (2005). Verordnung des SBFI über die berufliche Grundbildung Fachfrau Betreuung/Fachmann Betreuung. https://www. admin.ch/opc/de/classified-compilation/20051724/. Zugegriffen: 30. Juni 20.

Stern, Susanne, Schultheiss, Andrea, Fliedner, Juliane, Iten, Rolf, \& Christina, Felfe (2015). Analyse der Vollkosten und der Finanzierung von Krippenplätzen in Deutschland, Frankreich und Österreich im Vergleich zur Schweiz. Beiträge zur sozialen Sicherheit. Zürich, St. Gallen: Bundesamt für Sozialversicherungen. https://edudoc.ch/record/118119?ln=de. Zugegriffen 30. Juni 20.

Stern, Susanne, Gschwend, Eva, Iten, Rolf, \& Schwab Cammarano, Stephanie (2018). Kinderbetreuung und Erwerbstätigkeit: Was sich Eltern wünschen. Zürich: Jacobs Foundation. https://www.infras.ch/media/filer_public/c5/3d/c53dbe39-73db-497d-b675-775 024a43aca/schlussbericht_infras_gfs_bern_kinderbetreuung_und_erwerbstatigkeit_def. pdf. Zugegriffen: 30. Juni 20 ..

Taylor, Stephanie (2015). Discursive and Psychosocial? Theorising a Complex Contemporary Subject. Qualitative Research in Psychology, 12(1), 8-21.

Taylor, Shelley E., \& Fiske, Susan T. (1978). Salience, Attention, and Attribution: Top of the Head, in Leonard Berkowitz (Ed.), Advances in Experimental Social Psychology (Vol. 11, S. 249-288), Cambridge, Massachusetts: Academic Press.

Tennhoff, Wiebke (2018). Symbolische Väter, junge Wilde und professionelle Pädagogen. Diskursive Konstruktionen männlicher Geschlechtsidentität in Kindertageseinrichtungen (Dissertation). Universität St. Gallen. https://www.alexandria.unisg.ch/publications/ 257653. Zugegriffen 20. Juni 20.

Thorne, Barrie (1993). Gender Play: Girls and Boys in School. New Brunswick: Rutgers University Press.

Tyler, Melissa, \& Cohen, Laurie (2010). Spaces that Matter: Gender Performativity and Organizational Space. Organization Studies, 31(2), 175-198.

Van Lenning, Alkeline (2016). The Body As Crowbar: Transcending Or Stretching Sex? Feminist Theory, 5(1), 25-47.

Vogt, Franziska (2015). Bildung in der frühen Kindheit: "Bildung" und "pädagogische Qualität" als Auslöser von Angebotsausbau und Innovation. In Sandra Hupka-Brunner, Hans-Ulrich Grunder, Manfred Max Bergman, \& Christian Imdorf (Hrsg.), Qualität in der Bildung (S.13-27). Bad Heilbrunn: Julius Klinkhardt.

Wasserman, Varda, \& Frenkel, Michal (2015). Spatial Work in Between Glass Ceilings and Glass Walls: Gender-Class Intersectionality and Organizational Aesthetics. Organization Studies, 36(11), 1485-1505.

West, Candace, \& Zimmerman, Don H. (2009). Accounting for Doing Gender. Gender \& Society, 23(1), 112-122. 
West, Candace, \& Zimmerman, Don H. (1987). Doing Gender. Gender and Society, 1(2), $125-151$.

Westheuser, Linus (2015). Männer, Frauen und Stefan Hirschauer. Undoing gender zwischen Praxeologie und rhetorischer Modernisierung. GENDER - Zeitschrift für Geschlecht, Kultur und Gesellschaft, 3, 109-125.

Wetherell, Margaret (1998). Positioning and Interpretative Repertoires: Conversation Analysis and Post-Structuralism in Dialogue. Discourse \& Society, 9(3), 387-412.

Wetherell, Margaret (2008). Subjectivity or Psycho-Discursive Practices? Investigating Complex Intersectional Identities. Subjectivity, 22(1), 73-81.

Wetherell, Margaret (2010). The Field of Identity Studies. In Margareth Wetherell \& Chandra T. Mohanty (Eds.), The SAGE Handbook of Identities (pp. 3-26). London: Sage.

Wetherell, Margaret, \& Edley, Nigel (1999). Negotiating Hegemonic Masculinity: Imaginary Positions and Psycho-Discursive Practices. Feminism \& Psychology, 9(3), 335-356.

Wetherell, Margaret, \& Edley, Nigel (2014). A Discursive Psychological Framework for Analyzing Men and Masculinities. Psychology of Men \& Masculinity, 15(4), 355-364.

Wetterer, Angelika (2003). Rhetorische Modernisierung: Das Verschwinden der Ungleichheit aus dem zeitgenössischen Differenzwissen. In Gudrun-Axeli Knapp \& Angelika Wetterer (Hrsg.), Achsen der Differenz. Gesellschaftstheorie und feministische Kritik (S. 286-319). Münster: Westfälisches Dampfboot.

Wetterer, Angelika (2006). Ordentlich in Unordnung? Österreichische Zeitschrift für Soziologie, 31(4), 5-22.

Wickes, Rebecca, \& Emmison, Michael (2007). They are all 'Doing Gender' but are they all Passing? A Case Study of the Appropriation of a Sociological Concept. The Sociological Review, 55(2), 311-330.

Wilz, Sylvia M. (2008). Organisation: Die Debatte um, Gendered Organizations“ In R. Becker, \& B. Kortendieck (Hrsg.), Handbuch Frauen-und Geschlechterforschung (S. 505-511). Wiesbaden: VS Verlag für Sozialwissenschaften.

Witzel, Andreas (1989). Das Problemzentrierte Interview. In Gerd Jüttemann (Hrsg.), Qualitative Forschung in der Psychologie. Grundfragen, Verfahrensweisen, Anwendungsfelder (S. 227-255). Heidelberg: Asanger.

Wrana, Daniel (2012). Diesseits von Diskursen und Praktiken. Methodologische Bemerkungen zu einem Verhältnis. In Barbara Friebertshäuser (Hrsg.), Feld und Theorie: Herausforderungen erziehungswissenschaftlicher Ethnographie (S. 185-200). Opladen: Budrich.

Wrana, Daniel, \& Langer, Antje (2007). On the Edge of Discourse. Beyond the Differentiation of Discursive and Non-discursive Practices. Forum Qualitative Sozialforschung / Forum: Qualitative Social Research, 8(2), Art. 20.

Zietsma, Charlene, \& Lawrence, Thomas B. (2010). Institutional Work in the Transformation of an Organizational Field: The Interplay of Boundary Work and Practice Work. Administrative Science Quarterly, 55(2), 189-221.

Zinnecker, Jürgen (2000). Pädagogische Ethnographie. Zeitschrift für Erziehungswissenschaft, 3(3), 381-400. 
Open Access Dieses Kapitel wird unter der Creative Commons Namensnennung 4.0 International Lizenz (http://creativecommons.org/licenses/by/4.0/deed.de) veröffentlicht, welche die Nutzung, Vervielfältigung, Bearbeitung, Verbreitung und Wiedergabe in jeglichem Medium und Format erlaubt, sofern Sie den/die ursprünglichen Autor(en) und die Quelle ordnungsgemäß nennen, einen Link zur Creative Commons Lizenz beifügen und angeben, ob Änderungen vorgenommen wurden.

Die in diesem Kapitel enthaltenen Bilder und sonstiges Drittmaterial unterliegen ebenfalls der genannten Creative Commons Lizenz, sofern sich aus der Abbildungslegende nichts anderes ergibt. Sofern das betreffende Material nicht unter der genannten Creative Commons Lizenz steht und die betreffende Handlung nicht nach gesetzlichen Vorschriften erlaubt ist, ist für die oben aufgeführten Weiterverwendungen des Materials die Einwilligung des jeweiligen Rechteinhabers einzuholen.

(c) (1) 